D. Fujiwara

Nagoya Math. J.

Vol. 124 (1991), 61-97

\title{
THE STATIONARY PHASE METHOD WITH AN ESTIMATE OF THE REMAINDER TERM ON A SPACE OF LARGE DIMENSION
}

\author{
DAISUKE FUJIWARA
}

\section{Dedicated to the memory of Professor Kôsaku Yosida}

\section{§ 1. Introduction}

In discussing convergence of Feynman path integrals [2], we need a stationary phase method of oscillatory integrals over a space of large dimension. More precisely, we have to know how the remainder term behaves when the dimension of the space goes to $\infty$ (cf. [2], [3] and [5]). The aim of the present note is to give answer to this question under rather mild assumptions. Application to the Feynman path integrals is discussed in [3] and [5].

Oscillatory integral of a function $f(x), x \in \mathbb{R}^{k}$, is defined by the equality

$$
\int_{\mathbb{R}^{k}} f(x) d x=\lim _{\varepsilon \rightarrow 0} \int_{\mathrm{R}^{k}} e^{-\varepsilon|x|^{2}} f(x) d x .
$$

We consider the following oscillatory integrals

$$
I\left(\left\{t_{j}\right\}, S, a, \nu\right)\left(x_{L}, x_{0}\right)=\prod_{j=1}^{L}\left(\frac{\nu i}{2 \pi t_{j}}\right)^{d / 2} \tilde{\int}_{\mathrm{R}^{d(L-1)}} e^{-i_{\nu} S\left(x_{L}, \cdots, x_{0}\right)} a\left(x_{L}, \cdots, x_{0}\right) \prod_{j=1}^{L-1} d x_{j} .
$$

Here each $x_{j}, j=0, \cdots, L$, runs in $\mathbb{R}^{d}, \nu>1$ is a constant and $t_{j}, j=1$, $2, \cdots, L$, are positive constants.

Our assumption for the phase function $S\left(x_{L}, \cdots, x_{0}\right)$ is the following:

(H.1) $S\left(x_{L}, \cdots, x_{0}\right)$ is a real valued function of the form

$$
S\left(x_{L}, x_{L-1}, \cdots, x_{0}\right)=\sum_{j=1}^{L} S_{j}\left(t_{j}, x_{j}, x_{j-1}\right)
$$

where

Received October 2, 1990.

*) Research partially supported by Grant in Aid for General Scientific Research 01540108, The Ministry of Education. 


$$
S_{j}\left(t_{j}, x_{j}, x_{j-1}\right)=\frac{1}{2 t_{j}}\left|x_{j}-x_{j-1}\right|^{2}+t_{j} \omega_{j}\left(t_{j}, x_{j}, x_{j-1}\right), \quad j=1,2, \cdots, L .
$$

For any $m \geqq 2$ there exists a constant $\kappa_{m}>0$ independent of $j$ such that

$$
\max _{2 \leqq|\alpha|+|\beta| \leqq m} \sup _{x, y \in \mathbf{R}^{d}}\left|\partial_{x}^{\alpha} \partial_{y}^{\beta} \omega_{j}\left(t_{j}, x, y\right)\right| \leqq \kappa_{m} .
$$

(Since both $x$ and $y$ have $d$ components, both $\alpha$ and $\beta$ are multi-indices with $d$ components.)

We need a little more notations to write down our assumptions about the amplitude function. If $T_{L}=t_{1}+t_{2}+\cdots+t_{L}$ is small enough, the critical point $\left(x_{L-1}^{*}, x_{L-2}^{*}, \cdots, x_{1}^{*}\right)$ of the phase is the unique solution of

$$
\partial_{x_{j}} S_{j+1}\left(t_{j+1}, x_{j+1}^{*}, x_{j}^{*}\right)+\partial_{x_{j}} S_{j}\left(t_{j}, x_{j}^{*}, x_{j-1}^{*}\right)=0, \quad j=1,2, \cdots, L-1,
$$

where $x_{L}^{*}=x_{L}$ and $x_{0}^{*}=x_{0}$ (See $\S 2$ for the proof). We use the following notation

$$
a\left(\widetilde{x_{L}, x_{0}}\right)=a\left(x_{L}, x_{L-1}^{*}, \cdots, x_{1}^{*}, x_{0}\right) .
$$

Similarly, for any pair of integers $k, m$ with $k+1<m$ let $x_{k+1}^{*}, \cdots, x_{m-1}^{*}$ be the partial critical point, i.e.,

$$
\partial_{x_{j}} S_{j+1}\left(t_{j+1}, x_{j+1}^{*}, x_{j}^{*}\right)+\partial_{x_{j}} S_{j}\left(t_{j}, x_{j}^{*}, x_{j-1}^{*}\right)=0,
$$

for $j=k+1, \cdots, m-1$, where $x_{k}^{*}=x_{k}$ and $x_{m}^{*}=x_{m}$. Then we set

$$
a\left(x_{L}, \cdots, \widehat{x}_{m}, x_{k}, \cdots, x_{0}\right)=a\left(x_{L}, \cdots, x_{m}, x_{m-1}^{*}, \cdots, x_{k+1}^{*}, x_{k}, \cdots, x_{0}\right) .
$$

If $m=k+1$, we define

$$
a\left(x_{L}, x_{L-1}, \cdots,{\widetilde{x_{k+1}}}_{1}, \cdots, x_{0}\right)=a\left(x_{L}, \cdots, x_{k+1}, x_{k}, \cdots, x_{0}\right) .
$$

Our assumption for the amplitude function $a\left(x_{L}, \cdots, x_{0}\right)$ is the following:

(H.2) For any positive integer $K$ there exist positive constants $A_{K}$ and $X_{K}$ with the following properties:

(i) If $\left|\alpha_{j}\right| \leqq K$ for $j=0,1, \cdots, L$, then

$$
\sup _{\left(x_{L}, \cdots, x_{0}\right) \in \mathbf{R}^{L+1}}\left|\left(\prod_{j=0}^{L} \partial_{x_{j}}^{\alpha_{j}}\right) a\left(x_{L}, \cdots, x_{0}\right)\right| \leqq A_{K} X_{K}^{L} .
$$

(ii) For any sequence of positive integers

$$
j_{0}=0<j_{1}-1<j_{1}<j_{2}-1<\cdots<j_{s}<L, \quad s=1, \cdots, L-1,
$$


we have the estimate

$$
\left|\partial_{x_{0}}^{\alpha_{0}} \partial_{x_{j_{1}-1}}^{\alpha_{j_{1}-1}} \cdots \partial_{x_{j_{s}}}^{\alpha_{j_{s}} \partial_{x_{L}}^{\alpha} a} a\left({\overline{x_{L}, x_{j_{s}}}}_{x_{j_{s}-1}, x_{j_{s-1}}}, \cdots, \widetilde{x_{j_{1}-1}, x_{0}}\right)\right| \leqq A_{K} X_{K}^{s}
$$

as far as $\left|\alpha_{j}\right| \leqq K, j=0, j_{1}-1, j_{1}, \cdots, j_{s}, L$.

Our main result is

THEOREM 1. Under the assumptions (H.1) and (H.2) above there exists $a$ positive constant $\delta$ independent of $a$ and $L$ such that if $T_{L}=t_{1}+t_{2}+$ $\cdots+t_{L}<\delta$ then

$$
\begin{aligned}
& I\left(\left\{t_{j}\right\}, S, a, \nu\right)\left(x_{L}, x_{0}\right) \\
& \quad=\left(\frac{\nu i}{2 \pi T_{L}}\right)^{d / 2} \exp \left\{-i \nu S\left({\overrightarrow{x_{L}, x_{0}}}_{0}\right)\right\} \operatorname{det}\left(I+H^{-1} W\right)^{-1 / 2}\left(a\left(\vec{x}_{L}, x_{0}\right)+r\left(x_{L}, x_{0}\right)\right),
\end{aligned}
$$

where $r\left(x_{L}, x_{0}\right)$ satisfies the estimate: For any $K \geqq 0$ there exist positive constants $C_{K}$ and $M(K)$ such that if $\left|\alpha_{0}\right|,\left|\alpha_{L}\right| \leqq K$,

$$
\left|\partial_{x_{0}}^{\alpha_{0}} \partial_{x_{L}}^{\alpha} r\left(x_{L}, x_{0}\right)\right| \leqq A_{M(K)}\left(\prod_{j=1}^{L}\left(1+C_{K} X_{M(K)} \nu^{-1} t_{j}\right)-1\right) .
$$

Constants $\delta, C_{K}$ are independent of $a, L,\left\{t_{j}\right\},\left(x_{L}, x_{0}\right)$ and of $\nu$ but depend on the dimensionality $d$ of space $\mathbb{R}^{d}$ and $\left\{\kappa_{m}\right\} . M(K)$ depends only on $K$ and $d$. $H$ is the $d(L-1) \times d(L-1)$ matrix

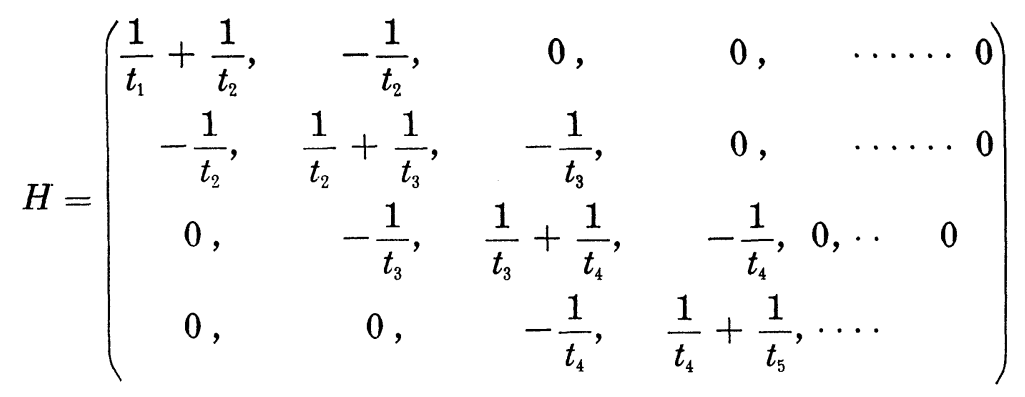

and $w$ is the Hessian matrix of $\sum_{j} t_{j} \omega_{j}\left(t_{j}, x_{j}, x_{j-1}\right)$ at the critical point $\left(x_{L-1}^{*}, \cdots, x_{1}^{*}\right)$.

In case $a=1$, we can prove a sharper estimate of the remainder term.

THEOREM 2. We assume that $a=1$ and (H.1). If $T_{L}<\delta$, then for any $K$ there exists a constant $C_{K}^{\prime}$ such that if $\left|\alpha_{0}\right|$ and $\left|\alpha_{L}\right| \leqq K$,

$$
\left|\partial_{x_{0}}^{\alpha_{0}} \partial_{x_{L}}^{\alpha_{L}} r\left(x_{L}, x_{0}\right)\right| \leqq \prod_{j=1}^{L}\left(1+C_{k}^{\prime} \nu^{-1} t_{j} T_{L}^{2}\right)-1
$$


The constant $\delta$ is the same as in Theorem 1 .

Remark. 1) We can easily see from the proof that the phase function and amplitude function are not necessarily infinitely differentiable. Both Theorems 1 and 2 are still valid with obvious modification if both functions are of class $C^{k}$ with sufficiently large $k$.

2) In the previous work [4], less sharp result was obtained.

The plan of the paper is as follows: Theorems 1 and 2 will be proved in the case $d=1$ in order to avoid excessive complexity of notations. In $\S 2$ we collect basic properties of both the critical point and the critical value of the phase function. In $\S 3$ we prove a key lemma which plays a fundamental role in this paper. This lemma, Lemma 3.1, may be of independent interest. In $\S 4$ Theorem 1 is proved. Theorem 2 is proved in $\S 5$.

Acknowledgements. In the author's original manuscript the exponent of $T_{L}$ on the right hand side of the estimate in Theorem 2 was 1. The referee kindly pointed out it is in fact 2 . The author wishes to express his sincere gratitude to the referee.

\section{§ 2. Phase functions}

In this section we discuss the followings:

(i) Unique existence of the critical point of $S$.

(ii) $S\left(\widetilde{x_{L}, x_{0}}\right)$ is of the form $\frac{\left|x_{L}-x_{0}\right|^{2}}{2 T_{L}}+T_{L} \omega^{\#}\left(x_{L}, x_{0}\right)$.

(iii) Some elementary facts related to the Hessian of $S$.

The critical point $x^{*}=\left(x_{L-1}^{*}, \cdots, x_{1}^{*}\right)$ of the phase function is given by the system of equations

$$
\begin{aligned}
& \frac{1}{t_{2}}\left(x_{1}^{*}-x_{2}^{*}\right)+\frac{1}{t_{1}}\left(x_{1}^{*}-x_{0}\right)+t_{2} \partial_{1} \omega_{2}\left(t_{2}, x_{2}^{*}, x_{1}^{*}\right)+t_{1} \partial_{1} \omega_{1}\left(t_{1}, x_{1}^{*}, x_{0}\right)=0, \\
& \frac{1}{t_{3}}\left(x_{2}^{*}-x_{3}^{*}\right)+\frac{1}{t_{2}}\left(x_{2}^{*}-x_{1}^{*}\right)+t_{3} \partial_{2} \omega_{3}\left(t_{3}, x_{3}^{*}, x_{2}^{*}\right)+t_{2} \partial_{2} \omega_{2}\left(t_{2}, x_{2}^{*}, x_{1}^{*}\right)=0, \\
& \frac{1}{t_{L}}\left(x_{L-1}^{*}-x_{L}\right)+\frac{1}{t_{L-1}}\left(x_{L-1}^{*}-x_{L-2}^{*}\right)+t_{L} \partial_{L-1} \omega_{L}\left(t_{L}, x_{L}, x_{L-1}^{*}\right) \\
& +t_{L-1} \partial_{L-1} \omega_{L-1}\left(t_{L-1}, x_{L-1}^{*}, x_{L-2}^{*}\right)=0 \text {. }
\end{aligned}
$$

Here and hereafter $\partial_{k}$ is the abbreviation of $\partial_{x_{k}}=\partial / \partial x_{k}$. 
The Hessian matrix of $S$ is equal to $H(L, 1)+W(L, 1 ; x)$, where

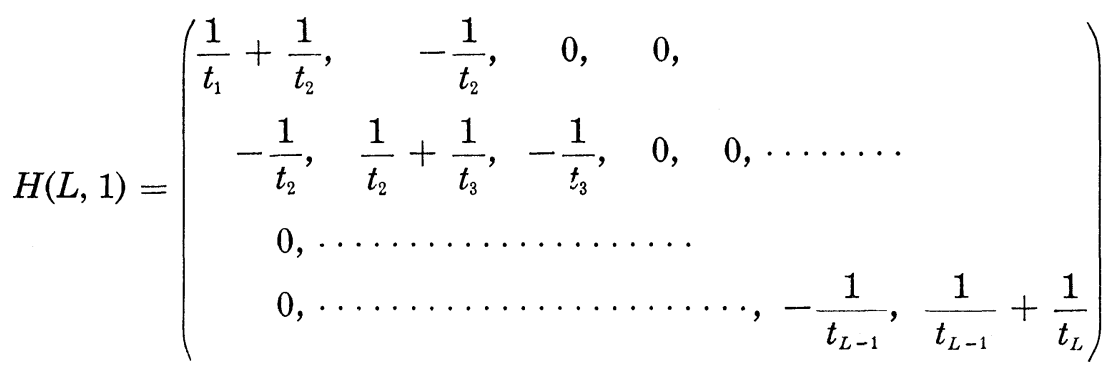

and

$$
W(L, 1 ; x)=\left(\begin{array}{lll}
t_{2} \partial_{1}^{2} \omega_{2}+t_{1} \partial_{1}^{2} \omega_{1}, \quad t_{2} \partial_{1} \partial_{2} \omega_{2}, & 0, \ldots \ldots, 0 \\
t_{2} \partial_{2} \partial_{1} \omega_{2}, & t_{3} \partial_{2}^{2} \omega_{3}+t_{2} \partial_{2}^{2} \omega_{2}, & t_{3} \partial_{2} \partial_{3} \omega_{3}, \quad 0, \ldots, 0 \\
\ldots \ldots \ldots \ldots \ldots \ldots \ldots \ldots &
\end{array}\right)
$$

We have

Proposition 2.1.

$$
\operatorname{det} H(L, 1)=\frac{T_{L}}{t_{1} t_{2} \cdots t_{L}} .
$$

Let $G(L, 1)$ be the inverse of $H(L, 1)$. Then its (ij) entry is

$$
\begin{array}{r}
g_{i j}=\frac{\left(t_{1}+\cdots+t_{i}\right)\left(t_{j+1}+\cdots+t_{L}\right)}{T_{L}}, \quad \text { if } 1 \leqq i \leqq j \leqq L-1, \\
\\
\quad \frac{\left(t_{1}+\cdots+t_{j}\right)\left(t_{i+1}+\cdots+t_{L}\right)}{T_{L}}, \quad \text { if } 1 \leqq j \leqq i \leqq L-1 .
\end{array}
$$

We use two norms $\|x\|_{\infty}=\max _{1 \leqq k \leqq L-1}\left|x_{k}\right|$ and $\|x\|_{1}=\sum_{j=1}^{L-1}\left|x_{j}\right|$ for any $x \in \mathbb{R}^{L-1}$. The next proposition is clear.

Proposition 2.2. For any $u \in \mathbb{R}^{L-1}$ we have

$$
\begin{aligned}
& \|W(L, 1 ; x) u\|_{1} \leqq 4 T_{L} \kappa_{2}\|u\|_{\infty}, \\
& \|G(L, 1) u\|_{\infty} \leqq \frac{T_{L}}{4}\|u\|_{1}
\end{aligned}
$$

and

$$
\|G(L, 1) W(L 1 ; x) u\|_{\infty} \leqq T_{L}^{2} \kappa_{2}\|u\|_{\infty} .
$$

Unique existence of the critical point of $S$ is given by 
Proposition 2.3. Assume that

$$
4 \kappa_{2} T_{L}^{2}<2^{-1}
$$

Then the critical point exists uniquely and satisfies the estimate:

$$
\left\|x^{*}-x^{0}\right\|_{\infty} \leqq \frac{T_{L}}{2} \sum_{k=1}^{L-1}\left|t_{k+1} \partial_{k} \omega_{k+1}\left(t_{k+1}, x_{k+1}^{0}, x_{k}^{0}\right)+t_{k} \partial_{k} \omega_{k}\left(t_{k}, x_{k}^{0}, x_{k-1}^{0}\right)\right|
$$

where

$$
x_{j}^{0}=\frac{t_{j+1}+\cdots+t_{L}}{T_{L}} x_{0}+\frac{t_{1}+\cdots+t_{j}}{T_{L}} x_{L}, \quad j=1, \cdots, L-1 .
$$

Proof. The critical point $x^{*}=\left(x_{L-1}^{*}, \cdots, x_{1}^{*}\right)$ is the fixed point of the $\operatorname{map}\left(x_{L-1}, \cdots, x_{1}\right)=x \rightarrow \Phi(x)=\left(y_{L-1}, \cdots, y_{1}\right)$, where

$$
\begin{aligned}
y_{j}=- & \sum_{k=1}^{L-1} g_{j k}\left\{t_{k+1} \partial_{k} \omega_{k+1}\left(t_{k+1}, x_{k+1}, x_{k}\right)+t_{k} \partial_{k} \omega_{k}\left(t_{k}, x_{k}, x_{k-1}\right)\right\} \\
& +g_{j 1} \frac{1}{t_{1}} x_{0}+g_{j L-1} \frac{1}{t_{L}} x_{L} .
\end{aligned}
$$

The norm of the differential map $D \Phi(x)=G(L, 1) W(L, 1 ; x)$ is less than $\kappa_{2} T_{L}^{2} \leqq 1 / 8$ with respect to the norm \|\|$_{\infty}$ because of (2.8) and (2.9). Therefore, the map $\Phi(x)$ is a contraction map, which guarantees unique existence of the fixed point. Usual construction by iteration of the fixed point gives that

$$
\left\|x^{*}-x^{0}\right\|_{\infty}<2\left\|\Phi\left(x^{0}\right)-x^{0}\right\|_{\infty} .
$$

We have $\Phi\left(x^{0}\right)-x^{0}=G(L, 1) \Omega\left(x^{0}\right)$, where $\Omega(x)=\left(\Omega_{1}(x), \cdots, \Omega_{L-1}(x)\right)$ and

$$
\Omega_{j}(x)=t_{j} \partial_{j} \omega_{j}\left(t_{j}, x_{j}, x_{j-1}\right)+t_{j+1} \partial_{j} \omega_{j+1}\left(t_{j+1}, x_{j+1}, x_{j}\right), \quad j=1, \cdots, L-1 .
$$

This and (2.7) yield that $\left\|\Phi\left(x^{0}\right)-x^{0}\right\|_{\infty}<\left(T_{L} / 4\right)\|\Omega\|_{1}$. This together with (2.12) proves the estimate (2.10). Proposition 2.3 has been proved.

Let $y$ and $z$ be points in $\mathbb{R}^{L-1}$ such that

$$
\begin{gathered}
y_{j}=g_{j 1}\left(t_{1}^{-1}-t_{1} \partial_{0} \partial_{1} \omega_{1}\left(t_{1}, x_{1}^{*}, x_{0}\right)\right), \quad j=1, \cdots, L-1, \\
z_{j}=g_{j L-1}\left(t_{L}^{-1}-t_{L-1} \partial_{L-1} \partial_{L} \omega_{L}\left(t_{L-1}, x_{L}, x_{L-1}^{*}\right)\right), \quad j=1, \cdots, L-1 .
\end{gathered}
$$

Then

$$
\|y\|_{\infty}, \quad\|z\|_{\infty} \leqq 1+\kappa_{2} T_{L}^{2}<\frac{9}{8}
$$


We consider the critical point as a function of $\left(x_{L}, x_{0}\right)$. Let $X=\partial_{0} x^{*}$ and $Y=\partial_{L} x^{*}$; let $D_{X} W\left(L, 1 ; x^{*}\right)$ and $D_{Y} W\left(L, 1 ; x^{*}\right)$ be derivatives at $x^{*}$ of matrix valued function $W(L, 1 ; x)$ in the direction $X$ and $Y$, respectively.

Proposition 2.4. We assume (2.9). Then we have

$$
\left\|\partial_{0} x^{*}-y\right\|_{\infty}, \quad\left\|\partial_{L-1} x^{*}-z\right\|_{\infty}<4 \kappa_{2} T_{L}^{2}<\frac{1}{2}
$$

and

$$
\left\|\partial_{0} x^{*}\right\|_{\infty}, \quad\left\|\partial_{L} x^{*}\right\|_{\infty}<1+4 \kappa_{2} T_{L}^{2}<\frac{3}{2} .
$$

For any integers $\alpha$ and $\beta$ there exists a positive constant $C_{\alpha \beta}$ such that

$$
\left\|D_{X}^{\alpha} D_{Y}^{\beta} W\left(L, 1 ; x^{*}\right) v\right\|_{1}<C_{\alpha \beta} T_{L}\|v\|_{\infty} .
$$

And for any $\alpha$ and $\beta$ with $\alpha+\beta \geqq 2$ we have, with some constant $C_{\alpha \beta}$,

$$
\left\|\partial_{0}^{\alpha} \partial_{L-1}^{\beta} x^{*}\right\|_{\infty} \leqq C_{\alpha \beta} T_{L}^{2} .
$$

The constants $C_{\alpha \beta}$ in (2.18) and (2.19) may depend on $T_{L}$ but are bounded if $T_{L}$ is bounded.

Proof. Since $x^{*}$ is the fixed point of $\Phi(x)$, we have that

$$
\partial_{0} x^{*}=\left(D \Phi\left(x^{*}\right)\right) \partial_{0} x^{*}+y .
$$

Since the norm of $D \Phi\left(x^{*}\right)$ is less than $\kappa_{2} T_{L}^{2}<1 / 8$, we have

$$
\left\|\partial_{0} x^{*}-y\right\|_{\infty}<2 \kappa_{2} T_{L}^{2}\|y\|_{\infty} \leqq 3 \kappa_{2} T_{L}^{2}<\frac{3}{8} .
$$

Therefore we have

$$
\left\|\partial_{0} x^{*}\right\|_{\infty} \leqq 1+4 \kappa_{2} T_{L}^{2}<\frac{3}{2}
$$

Similarly we can prove estimate for $\partial_{L} x^{*}$. (2.16) and (2.17) are proved.

Next we prove (2.18). It follows from (2.16) and (2.17) that for each pair of indices $\alpha$ and $\beta$ there exist $\mathbb{R}^{L-1}$ valued functions $a^{\alpha \beta}\left(x_{L}, x_{0}\right)$, $b^{\alpha \beta}\left(x_{L}, x_{0}\right)$ and $c^{\alpha \beta}\left(x_{L}, x_{0}\right)$ satisfying

$$
\begin{aligned}
\left(D_{X}^{\alpha} D_{Y}^{\beta} W\left(L, 1 ; x^{*}\right)\right)_{j k} & =0, & & \text { for }|k-j|>1, \\
& =t_{j} a_{j}^{\alpha \beta}\left(x_{L}, x_{0}\right), & & \text { for } k=j-1, \\
& =t_{j} b_{j}^{\alpha \beta}\left(x_{L}, x_{0}\right)+t_{j+1} c_{j}^{\alpha \beta}\left(x_{L}, x_{0}\right), & & \text { for } k=j, \\
& =t_{j+1} a_{j+1}^{\alpha \beta}\left(x_{L}, x_{0}\right), & & \text { for } k=j+1 .
\end{aligned}
$$


These functions $a^{\alpha \beta}\left(x_{L}, x_{0}\right), b^{\alpha \beta}\left(x_{L}, x_{0}\right)$ and $c^{\alpha \beta}\left(x_{L}, x_{0}\right)$ may depend also on $t_{1}, \cdots, t_{L}$ but remain uniformly bounded in the space $\mathscr{B}\left(\mathbb{R}_{x_{L}} \times \mathbb{R}_{x_{0}}\right)$ as far as (2.9) holds. This proves (2.18).

We shall prove the estimate for $\partial_{0} \partial_{L} x^{*}$. It satisfies

$$
\partial_{L} \partial_{0} x^{*}=D \Phi\left(x^{*}\right) \partial_{L} \partial_{0} x^{*}+G(L, 1) D_{Y} W\left(L, 1 ; x^{*}\right) \partial_{0} x^{*}+\partial_{L} y .
$$

Thus using (2.18), we have

$$
\begin{aligned}
\left\|\partial_{L} \partial_{0} x^{*}\right\|_{\infty} & \leqq 2\left(\left\|G(L, 1) D_{Y} W\left(L, 1 ; x^{*}\right) \partial_{0} x^{*}\right\|_{\infty}+\left\|\partial_{L} y\right\|_{\infty}\right) \\
& \leqq C T_{L}^{2}\left\|\partial_{0} x^{*}\right\|_{\infty}+C T_{L}^{2} \leqq C T_{L}^{2}
\end{aligned}
$$

Here and hereafter we denote simply by $C$ various constants which may be different from one occasion to another. Other higher derivatives of $x^{*}$ will be estimated similarly. Proposition is proved.

Since $\partial_{0} x^{*}$ satisfies $(2.20)$ and $D \Phi\left(x^{*}\right)=G(L, 1) W\left(L, 1 ; x^{*}\right)$,

$$
\partial_{0} x^{*}=G(L, 1) Z\left(x_{L}, x_{0}\right)+y,
$$

where $Z\left(x_{L}, x_{0}\right)=W\left(L, 1 ; x^{*}\right) \partial_{0} x^{*}$. Using (2.6), we have

$$
\|Z\|_{1}=\left\|W\left(L, 1 ; x^{*}\right) \partial_{0} x^{*}\right\|_{1} \leqq 4 \kappa_{2} T_{L}\left\|\partial_{0} x^{*}\right\|_{\infty}<6 \kappa_{2} T_{L} .
$$

Moreover, the $j$-th component of $Z\left(x_{L}, x_{0}\right)$ is of the form

$$
Z_{j}\left(x_{L}, x_{0}\right)=t_{j} \xi_{j}\left(x_{L}, x_{0}\right)+t_{j+1} \eta_{j}\left(x_{L}, x_{0}\right), \quad j=1, \cdots, L-1,
$$

where $\left\{\xi_{j}\right\}$ and $\left\{\eta_{j}\right\}$ are functions, which may depend on $t_{1}, \cdots, t_{L}$ but bounded in $\mathscr{B}\left(\mathbb{R}_{x_{L}} \times \mathbb{R}_{x_{0}}\right)$. It follows from this that

$$
T_{L}^{-1} \sum_{j=1}^{L-1} Z_{j}\left(x_{L}, x_{0}\right) \text { remains bounded in } \mathscr{B}\left(\mathbb{R}_{x_{L}} \times \mathbb{R}_{x_{0}}\right) \text {. }
$$

Next we consider the second derivatives of the critical value $S\left(\overline{x_{L}, x_{0}}\right)$.

Proposition 2.5. We assume (2.9). Then $S\left(x_{L}, x_{0}\right)$ is of the following form:

$$
S\left(\widetilde{x_{L}, x_{0}}\right)=\frac{\left|x_{L}-x_{0}\right|^{2}}{2 T_{L}}+T_{L} \omega^{\sharp}\left(x_{L}, x_{0}\right) .
$$

Here $\omega^{\sharp}\left(x_{L}, x_{0}\right)$ is a function, which may depend also on $t_{1}, \cdots, t_{L}$ but remains bounded in $\mathscr{B}\left(\mathbb{R}_{x_{L}} \times \mathbb{R}_{x_{0}}\right)$, satisfying the estimate

$$
\max _{2 \leqq|\alpha|+|\beta| \leqq m} \sup _{x_{L}, x_{0}}\left|\partial_{x_{0}}^{\alpha} \partial_{x_{L}}^{\beta} \omega^{\sharp}\left(x_{L}, x_{0}\right)\right| \leqq \kappa_{m}^{\sharp},
$$


where $\kappa_{m}^{*}$ is a constant depending only on $\kappa_{j}, 2 \leqq j \leqq m$. We can choose

$$
\kappa_{2}^{\#}=10 \kappa_{2} \text {. }
$$

Proof. Since $x^{*}$ is the critical point of $S$, we have

$$
\begin{aligned}
\partial_{0} S\left(\widetilde{x_{L}, x_{0}}\right) & =\left.\partial_{0} S\left(x_{L}, x_{L-1}, \cdots, x_{1}, x_{0}\right)\right|_{x_{L-1}=x_{L-1}^{*}, \cdots, x_{1}=x_{1}^{*}} \\
& =\left(\partial_{0} S_{1}\right)\left(x_{1}^{*}, x_{0}\right),
\end{aligned}
$$

where we abbreviated $S_{1}\left(t_{1}, x_{1}, x_{0}\right)$ simply by $S_{1}\left(x_{1}, x_{0}\right)$. This implies that

$$
\partial_{0}^{2} S\left(\widetilde{x_{L}, x_{0}}\right)=\left(\partial_{0}^{2} S_{1}\right)\left(x_{1}^{*}, x_{0}\right)+\left(\partial_{1} \partial_{0} S_{1}\right)\left(x_{1}^{*}, x_{0}\right) \partial_{0} x_{1}^{*} .
$$

We have from (2.21) and (2.13) that

$$
\partial_{0} x_{1}^{*}-\frac{t_{2}+\cdots+t_{L}}{T_{L}}=t_{1} T_{L} h\left(x_{L}, x_{0}\right)
$$

where

$$
\begin{aligned}
h\left(x_{L}, x_{0}\right)= & -T_{L}^{-2} t_{1}\left(t_{2}+\cdots+t_{L}\right) \partial_{0} \partial_{1} \omega_{1}\left(t_{1}, x_{1}^{*}, x_{0}\right) \\
& +T_{L}^{-2} \sum_{j=1}^{L-1}\left(t_{j+1}+\cdots+t_{L}\right) Z_{j}\left(x_{L}, x_{0}\right)
\end{aligned}
$$

depends also on $t_{1}, \cdots, t_{L}$ but we used abbreviation. Since $T_{L}^{-1} \sum_{j=1}^{L-1} Z_{j}\left(x_{L}, x_{0}\right)$ is bounded in $\mathscr{B}\left(\mathbb{R}_{x_{L}} \times \mathbb{R}_{x_{0}}\right), h\left(x_{L}, x_{0}\right)$ remains bounded in $\mathscr{B}\left(\mathbb{R}_{x_{L}} \times \mathbb{R}_{x_{0}}\right)$ uniformly with respect to $t_{1}, \cdots, t_{L}$. In particular, (2.22) yields that

$$
\left|h\left(x_{L}, x_{0}\right)\right| \leqq T_{L}^{-1}\|Z\|_{1}+\kappa_{2}<7 \kappa_{2} .
$$

Hence we have

$$
\partial_{x_{0}}^{2} S\left(\widetilde{x_{L}, x_{0}}\right)=\frac{1}{T_{L}}+T_{L} \psi\left(x_{L}, x_{0}\right)
$$

where

$$
\begin{aligned}
\psi\left(x_{L}, x_{0}\right)= & -h\left(x_{L}, x_{0}\right)+\frac{t_{1}}{T_{L}} \partial_{0}^{2} \omega_{1}\left(x_{1}^{*}, x_{0}\right) \\
& +\left(\frac{t_{1}\left(t_{2}+\cdots+t_{L}\right)}{T_{L}^{2}}+t_{1}^{2} h\left(x_{L}, x_{0}\right)\right) \partial_{0} \partial_{1} \omega_{1}\left(x_{1}^{*}, x_{0}\right)
\end{aligned}
$$

remains bounded in the space $\mathscr{B}\left(\mathbb{R}_{x_{L}} \times \mathbb{R}_{x_{0}}\right)$. Moreover by definition $\left|\partial_{0}^{2} \omega_{1}\left(x_{1}^{*}, x_{0}\right)\right| \leqq \kappa_{2}$ and $\left|\partial_{0} \partial_{1} \omega_{1}\left(x_{1}^{*}, x_{0}\right)\right| \leqq \kappa_{2}$. And (2.28) and (2.9) imply that $t_{1}^{2}\left|h\left(x_{L}, x_{0}\right)\right|<1$. Therefore

$$
\left|\psi\left(x_{L}, x_{0}\right)\right| \leqq 10 \kappa_{2}
$$


Similar discussions hold for other derivatives of $S\left(\overline{x_{L}, x_{0}}\right)$. Therefore, we have proved Proposition 2.5.

Finally we discuss Hessian determinant of $S$.

Proposition 2.6. Let $\phi(x, y)$ be a real valued $C^{\infty}$-function of $(x, y) \in$ $\mathbb{R}^{m} \times \mathbb{R}^{n}$. Let $y^{\sharp}: \mathbb{R}^{m} \ni x \rightarrow y^{\sharp}(x) \in \mathbb{R}^{n}$ be a $C^{\infty}$-map such that

$$
\partial_{y} \phi\left(x, y^{\sharp}(x)\right)=0 \quad \text { for any } x \in \mathbb{R}^{m} .
$$

We assume that

$$
\left.\operatorname{det} \operatorname{Hess}_{y} \phi(x, y)\right|_{y=y^{\sharp}(x)} \neq 0
$$

and that the map $\phi^{\sharp}: \mathbb{R}^{m} \ni x \rightarrow \phi\left(x, y^{\sharp}(x)\right) \in \mathbb{R}$ has a critical point $x^{*}$, i.e.,

$$
\partial_{x} \phi^{\sharp}\left(x^{*}\right)=0 \text {. }
$$

Then $\left(x^{*}, y^{*}\right)=\left(x^{*}, y^{\sharp}\left(x^{*}\right)\right)$ is a critical point of $\phi(x, y)$. Moreover we have the product formula of Hessian determinant:

$$
\operatorname{det} \operatorname{Hess}_{\left(x^{*}, y^{*}\right)} \phi=\left.\left(\operatorname{det} \operatorname{Hess}_{x^{*}} \phi^{\sharp}\right)\left(\operatorname{det} \operatorname{Hess}_{y} \phi(x, y)\right)\right|_{(x, y)=\left(x^{*}, y^{*}\right)} .
$$

Proof. We have, from (2.29),

$$
\partial_{y} \phi\left(x^{*}, y^{*}\right)=0 \text {. }
$$

On the other hand we have, from (2.31), that

$$
\partial_{x} \phi\left(x^{*}, y^{*}\right)+\partial_{y} \phi\left(x^{*}, y^{*}\right) \partial_{x} y^{*}\left(x^{*}\right)=\partial_{x} \phi^{\sharp}\left(x^{*}\right)=0 .
$$

This and (2.33) gives that $\partial_{x} \phi\left(x^{*}, y^{*}\right)=0$. Therefore, $\left(x^{*}, y^{*}\right)$ is a critical point of $\phi$.

We have

$$
\operatorname{Hess}_{\left(x^{*}, y^{*}\right)} \phi=\left.\left(\begin{array}{cc}
\partial_{x}^{2} \phi, & \partial_{y} \partial_{x} \phi \\
\partial_{x} \partial_{y} \phi, & \partial_{y}^{2} \phi
\end{array}\right)\right|_{(x, y)=\left(x^{*}, y^{*}\right)} .
$$

On the other hand we have

$$
\begin{aligned}
\operatorname{Hess}_{x} \phi^{\#} & =\partial_{x}^{2} \phi\left(x^{*}, y^{*}\right)+\partial_{y} \partial_{x} \phi\left(x^{*}, y^{*}\right) \partial_{x} y^{\sharp}\left(x^{*}\right) \\
& =\partial_{x}^{2} \phi\left(x^{*}, y^{*}\right)-\partial_{y} \partial_{x} \phi\left(x^{*}, y^{*}\right) \partial_{y}^{2} \phi\left(x^{*}, y^{*}\right)^{-1} \partial_{x} \partial_{y} \phi\left(x^{*}, y^{*}\right),
\end{aligned}
$$

because

$$
\partial_{y}^{2} \phi\left(x, y^{\sharp}(x)\right) \partial_{x} y^{\sharp}(x)+\partial_{x} \partial_{y} \phi\left(x, y^{\sharp}(x)\right)=0 \text {. }
$$

Therefore the next Lemma proves (2.32). Proposition 2.6 is proved. 
Lemma 2.7. Let $A$ be a $(m+n) \times(m+n)$ matrix. We write

$$
A=\left(\begin{array}{cc}
B, & C \\
{ }^{t} C, & D
\end{array}\right)
$$

where $B$ is an $m \times m$ matrix, $C$ is an $m \times n$ matrix and $D$ is an $n \times n$ regular matrix. Then we have

$$
\operatorname{det}(A)=\operatorname{det}(D) \operatorname{det}\left(B-C D^{-1 t} C\right) .
$$

Proof. Take the determinant of the following matrix identity:

$$
\left(\begin{array}{cc}
B, & C \\
{ }^{t} C, & D
\end{array}\right)\left(\begin{array}{cc}
I, & 0 \\
-D^{-1}{ }^{t} C, & I
\end{array}\right)=\left(\begin{array}{cc}
B-C D^{-1 t} C, & C \\
0, & D
\end{array}\right)
$$

Let $x_{1}^{*}$ be the critical point with respect to $x_{1}$ of $S_{2}\left(x_{2}, x_{1}\right)+S_{1}\left(x_{1}, x_{0}\right)$. We define a function $D\left(S_{2}+S_{1} ; x_{2}, x_{1}\right)$ from the hessian determinant at $x_{1}^{*}$ in the following way:

$$
\operatorname{det} \operatorname{Hess}_{x^{*}}\left(S_{2}+S_{1}\right)=\frac{t_{1}+t_{2}}{t_{1} t_{2}} D\left(S_{2}+S_{1} ; x_{2}, x_{0}\right)
$$

Let $k<m$ be two positive integers. Then we define $\left(x_{m-1}^{*}, \cdots, x_{k+1}^{*}\right)$ as the partial critical point, i.e.,

$$
\partial_{j} S_{j+1}\left(x_{j+1}^{*}, x_{j}^{*}\right)+\partial_{j} S\left(x_{j}^{*}, x_{j-1}^{*}\right)=0, \quad j=k+1, \cdots, m-1 .
$$

Here $x_{m}^{*}=x_{m}$ and $x_{k}^{*}=x_{k}$. We denote the critical level by $S_{m, k+1}^{\sharp}\left(x_{m}, x_{k}\right)$, i.e.,

$$
S_{m, k+1}^{\#}\left(x_{m}, x_{k}\right)=S_{m}\left(t_{m}, x_{m}, x_{m-1}^{*}\right)+\cdots+S_{k+1}\left(t_{k+1}, x_{k+1}^{*}, x_{k}\right) .
$$

As a consequence of Proposition 2.5 we can write

$$
S_{m, k+1}^{\sharp}\left(x_{m}, x_{k}\right)=\frac{\left(x_{m}-x_{k}\right)^{2}}{2\left(t_{k+1}+\cdots+t_{m}\right)}+\left(t_{k+1}+\cdots+t_{m}\right) \omega_{m, k+1}^{\sharp}\left(x_{m}, x_{k}\right) .
$$

We define $D\left(x_{m}, x_{k}\right)$ by

$$
\operatorname{det}\left(\operatorname{Hess}_{\left(x_{m-1}^{*}, \cdots, x_{k+1}^{*}\right)}\left(S_{m}+\cdots+S_{k+1}\right)\right)=\frac{t_{k+1}+\cdots+t_{m}}{t_{k+1} \cdots t_{m}} D\left(x_{m}, x_{k}\right) .
$$

If $m=1$ and $k=1$ then we set $S_{1,1}^{\#}\left(x_{1}, x_{0}\right)=S_{1}\left(t_{1}, x_{1}, x_{0}\right)$.

Proposition 2.8. We have

$$
D\left(x_{L}, x_{0}\right)=\left.\left(\prod_{k=2}^{L} D\left(S_{k}+S_{k-1,1}^{\#} ; x_{k}, x_{0}\right)\right)\right|_{\left(x_{L-1}, \ldots, x_{1}\right)=\left(x_{L-1}^{*}, \ldots, x_{1}^{*}\right)} .
$$


Proof. We prove (2.38) by induction on $L$. The case $L=2$ is clear.

By induction hypothesis the Hessian determinant of $S_{L-1}+\cdots+S_{1}$ at the critical point $x^{\#}=\left(x_{L-2}^{\#}\left(x_{L-1}, x_{0}\right), \cdots, x_{1}^{\#}\left(x_{L-1}, x_{0}\right)\right)$ with respect to $x_{L-2}, \cdots, x_{1}$ equals

$$
\left.\frac{t_{1}+\cdots+t_{L-1}}{t_{1} \cdots t_{L-1}}\left(\prod_{k=2}^{L-1} D\left(S_{k}+S_{k-1,1}^{*} ; x_{k}, x_{0}\right)\right)\right|_{x_{j}=x_{j}^{\sharp}\left(x_{L-1}, x_{0}\right), j=1, \cdots, L-2} .
$$

So Proposition 2.6 gives that

$$
\begin{aligned}
& \frac{t_{1}+\cdots+t_{L}}{t_{1} \cdots t_{L}} D\left(x_{L}, x_{0}\right) \\
& =\left.\operatorname{det} \operatorname{Hess}_{x_{L-1}^{*}}\left(S_{L}+S_{L-1,1}^{\#}\right) \operatorname{det} \operatorname{Hess}_{\left(x_{L-2}^{*}, \cdots, x_{1}^{\sharp}\right)}\left(S_{L-1}+\cdots+S_{1}\right)\right|_{x_{L-1}=x_{L-1}^{*}} \\
& =\frac{t_{L}+\cdots+t_{1}}{t_{L}\left(t_{L-1}+\cdots+t_{1}\right)} D\left(S_{L}+S_{L-1,1}^{\#} ; x_{L-1}, x_{0}\right) \\
& \quad \quad \times\left.\frac{t_{1}+\cdots+t_{L-1}}{t_{1} \cdots t_{L-1}}\left(\prod_{k=2}^{L-1} D\left(S_{k}+S_{k-1,1}^{\#} ; x_{k}, x_{0}\right)\right)\right|_{x_{j=x_{j}^{*}}} .
\end{aligned}
$$

We have proved (2.38) for $L$ and Proposition is proved.

Proposition 2.9. We have

$$
D\left(S_{2}+S_{1} ; x_{2}, x_{0}\right)=1+t_{1} t_{2} g\left(x_{2}, x_{0}\right) .
$$

Here $g\left(x_{2}, x_{0}\right)$ remains bounded in $\mathscr{B}\left(\mathbb{R}_{x_{2}} \times \mathbb{R}_{x_{0}}\right)$.

Proof.

$$
\operatorname{Hess}_{x_{1}}\left(S_{2}+S_{1}\right)=\frac{t_{1}+t_{2}}{t_{1} t_{2}}\left(1+\frac{t_{1} t_{2}}{t_{1}+t_{2}}\left(t_{1} \partial_{1}^{2} \omega_{1}+t_{2} \partial_{1}^{2} \omega_{2}\right)\right) .
$$

This proves Proposition.

Proposition 2.10. Assume that (2.9) holds. We write

$$
\operatorname{det} \operatorname{Hess}_{x^{*}} S\left(x_{L}, x_{0}\right)=\frac{T_{L}}{t_{1} t_{2} \cdots t_{L}} D\left(x_{L}, x_{0}\right) .
$$

Then

$$
D\left(x_{L}, x_{0}\right)=1+T_{L}^{2} q\left(x_{L}, x_{0}\right),
$$

where $q\left(x_{L}, x_{0}\right)$ may depend also on $t_{1}, \cdots, t_{L}$ but remains bounded in the space $\mathscr{B}(\mathbb{R} \times \mathbb{R})$ uniformly with respect to $t_{1}, \cdots, t_{L}$.

Proof. We have from Proposition 2.8 that 


$$
D\left(x_{L}, x_{0}\right)=\left.\left(\prod_{k=2}^{L} D\left(S_{k}+S_{k+1,1}^{\sharp} ; x_{k}, x_{0}\right)\right)\right|_{\left(x_{L-1}, \ldots, x_{1}\right)=\left(x_{L-1}^{*}, \ldots, x_{1}^{*}\right)} .
$$

We can apply the previous proposition. Then we have

$$
D\left(x_{L}, x_{0}\right)=\prod_{k=2}^{L}\left(1+t_{k} T_{k-1} g_{k}\left(x_{L}, x_{0}\right)\right)
$$

where $g_{k}\left(x_{L}, x_{0}\right)$ are bounded in the space $\mathscr{B}(\mathbb{R} \times \mathbb{R})$. Proposition is proved.

\section{§ 3. Key Lemma}

The aim of the section is to prove the following Lemma 3.1, which plays an important role in the proof of the main results. In the present section assumption (H.2) is not needed. Instead we require the following assumption about the amplitude function:

(H.3) For any $K \geqq 0$ there exists a positive constant $A_{K}$ such that

$$
\max _{\alpha} \sup _{x_{L-1}, \cdots, x_{1}}\left|\partial_{x_{L}}^{\alpha_{L}} \partial_{x_{L-1}}^{\alpha_{L-1}} \cdots \partial_{x_{0}}^{\alpha_{0}} a\left(x_{L}, \cdots, x_{0}\right)\right|<A_{K},
$$

where $\max$ is taken with respect to multi-indices $\left(\alpha_{L}, \cdots, \alpha_{0}\right)$ satisfying $\left|\alpha_{j}\right| \leqq K, j=0,1, \cdots, L$.

LEMMA 3.1. We assume the hypothesis (H.1) for the phase function and hypothesis (H.3) above for the amplitude function. Then there exists a positive constant $\delta>0$ such that $I\left(\left\{t_{j}\right\}, S, a, \nu\right)\left(x_{L}, x_{0}\right)$ can be written as

$$
I\left(\left\{t_{j}\right\}, S, a, \nu\right)\left(x_{L}, x_{0}\right)=\left(\frac{\nu i}{2 \pi T_{L}}\right)^{1 / 2} \exp \left\{-i \nu S\left(\overline{x_{L}, x_{0}}\right)\right\} b\left(x_{L}, x_{0}\right),
$$

as far as $T_{L}=t_{1}+t_{2}+\cdots+t_{L}<\delta$. For any $m \geqq 0$ there exist constants $C_{m}$ and $K(m)$ such that if $\left|\alpha_{0}\right| \leqq m,\left|\alpha_{L}\right| \leqq m$,

$$
\left|\partial_{x_{L}}^{\alpha_{L}} \partial_{x_{0}}^{\alpha_{0}} b\left(x_{L}, x_{0}\right)\right| \leqq C_{m}^{L} \max _{\beta} \sup _{x_{L-1}, \cdots, x_{1}}\left|\partial_{x_{L}}^{\beta_{L}} \partial_{x_{L-1}}^{\beta_{L-1}} \cdots \partial_{x_{0}}^{\beta_{0}} a\left(x_{L}, \cdots, x_{0}\right)\right|,
$$

where $\max$ is taken with respect to all $\left(\beta_{L}, \cdots, \beta_{0}\right)$ satisfying $\beta_{0} \leqq \alpha_{0}, \beta_{L} \leqq \alpha_{L}$ and $\left|\beta_{j}\right| \leqq K(m)$. Here constants $K(m)$ and $C_{m}$ do not depend on $L, \nu$ and a. We can choose $K(m)=4 m+17+6$.

Proof of basic Lemma 3.1 will be given after Lemma 3.6. Before that, we collect preparatory facts. Most of them are well known but we will write them down for the convenience of the reader.

From now on we let $E=(i \nu / 2 \pi)$ for the sake of brevity of notation. 
The following Lemma is found in Kumanogo [6],

Lemma 3.2 (Kumanogo [6]). Let

$$
\begin{aligned}
J(\mu, a) \psi\left(x_{L}\right)= & \left(\frac{\mu}{2 \pi}\right)^{L} \int_{\mathrm{R}^{2 L}} \exp \left\{-i \mu \sum_{j=1}^{L} \xi_{j}\left(x_{j+1}-x_{j}\right)\right\} \\
& \times a\left(x_{L+1}, \xi_{L}, \cdots, \xi_{1}, x_{1}\right) \psi\left(x_{1}\right) \prod_{j=1}^{L} d \xi_{j} d x_{j} .
\end{aligned}
$$

Then there exists a function $U(a)\left(x_{L+1}, \xi_{1}, x_{1}\right)$ such that

$$
J(\mu, a) \psi\left(x_{L+1}\right)=\left(\frac{\mu}{2 \pi}\right) \int_{R^{2}} \exp \left\{-i \mu\left(x_{L+1}-x_{1}\right) \xi_{1}\right\} U(a)\left(x_{L+1}, \xi_{1}, x_{1}\right) \psi\left(x_{1}\right) d x_{1} d \xi_{1} .
$$

We have

$$
\begin{gathered}
\partial_{x_{L+1}} U(a)\left(x_{L+1}, \xi_{1}, x_{1}\right)=U\left(\sum_{j=1}^{L+1} \partial_{x_{j}} a\right)\left(x_{L+1}, \xi_{1}, x_{1}\right), \\
\partial_{\xi_{1}} U(a)\left(x_{L+1}, \xi_{1}, x_{1}\right)=U\left(\sum_{j=1}^{L+1} \partial_{\xi_{j}} a\right)\left(x_{L+1}, \xi_{1}, x_{1}\right),
\end{gathered}
$$

and

$$
\partial_{x_{1}} U(a)\left(x_{L+1}, \xi_{1}, x_{1}\right)=U\left(\partial_{x_{1}} a\right)\left(x_{L+1}, \xi_{1}, x_{1}\right) .
$$

Moreover, there exists a constant $C_{0}$ independent of $\mu, L$ and of a such that

$$
\sup \left|U(a)\left(x_{L+1}, \xi_{1}, x_{1}\right)\right| \leqq C_{0}^{L}\|a\|_{3}
$$

where

$$
\|a\|_{k}=\max _{\left|\alpha_{j}\right|,\left|\beta_{j}\right| \leqq k} \sup \left|\left(\prod_{j=1}^{L} \partial_{x_{j}}^{\alpha_{j}} \partial_{\xi_{j}}^{\beta_{j}}\right) a\left(x_{L+1}, \xi_{L}, x_{L}, \cdots, \xi_{1} x_{1}\right)\right| .
$$

Proof is found in Kumanogo [6]. A simple corollary is

CoROLlaRY 3.3. For any $m \geqq 0$ there are constants $C_{m}$ and $K_{1}(m)$ such that if $\left|\alpha_{L+1}\right|,\left|\beta_{1}\right|,\left|\alpha_{1}\right| \leqq m$,

$$
\sup \left|\partial_{x_{L+1}}^{\alpha_{L+1}} \partial_{\xi_{1}}^{\beta_{1}} \partial_{x_{1}}^{\alpha_{1}} U(a)\left(x_{L+1}, \xi_{1}, x_{1}\right)\right| \leqq C_{m}(L+1)^{2 m} C_{1}^{L+1}\|a\|_{K_{1}(m)} .
$$

We can choose $K_{1}(m)=2 m+3$.

Let $S(t, x, y)=(1 / 2 t)|x-y|^{2}+t \omega(t, x, y)$ and let $a(x, y)$ be in the space $\mathscr{B}\left(\mathbb{R}_{x} \times \mathbb{R}_{y}\right)$, then for any $f(y) \in C_{0}^{\infty}(\mathbb{R})$ we set

$$
\mathrm{Op}(t, S, a, \nu) f(x)=\left(\frac{E}{t}\right)^{1 / 2} \int_{\mathrm{R}} e^{-i \nu S(t, x, y)} a(x, y) f(y) d y .
$$


We assume as in $\S 1$ that

$$
\max _{2 \leqq|\alpha|+|\beta| \leqq m} \sup _{x, y \in \mathbf{R}}\left|\partial_{x}^{\alpha} \partial_{y}^{\beta} \omega(x, y)\right| \leqq \kappa_{m} .
$$

If $8|t|^{2} \kappa_{2}<1$ then $\operatorname{Op}(t, S, a, \nu)$ defines a bounded linear operator on $L^{2}(\mathbb{R})$ (cf. [1]). Its adjoint $\mathrm{Op}(t, S, a, \nu)^{*}$ is of the form

$$
\mathrm{Op}(t, S, a, \nu)^{*} f(x)=\left(\frac{E}{t}\right)^{1 / 2} \int_{\mathrm{R}} e^{i \nu S(t, z, x)} \overline{a(z, x)} f(z) d z
$$

Lemma 3.4. Assume that $S(t, x, y)$ is as above. Then there exists a positive constant $\delta_{1}=\delta_{1}\left(\left\{\kappa_{m}\right\}\right)$ depending only on dimensionality of the space and $\left\{\kappa_{m}\right\}_{m}$ such that if $|t| \leqq \delta_{1}$ then $\operatorname{Op}(t, S, 1, \nu)^{-1}$ exists and is of the form

$$
\mathrm{Op}(t, S, 1, \nu)^{-1}=\mathrm{Op}(t, S, 1+t p, \nu)^{*},
$$

where $p(t, x, y)$ satisfies the estimate: For any multi-indices $\alpha$ and $\beta$ there exists a positive constant $C_{\alpha \beta}$ independent of $t, \nu$ such that

$$
\left|\partial_{x}^{\alpha} \partial_{y}^{\beta} p(t, x, y)\right| \leqq C_{\alpha \beta} .
$$

Proof is given in [3].

Let $S_{i}\left(t_{i}, x, y\right)=\left(1 /\left(2 t_{i}\right)\right)|x-y|^{2}+t_{i} \omega_{i}\left(t_{i}, x, y\right), i=1,2$, be phase functions and $a(x, y, z)$ be an amplitude function as in $\S 1$. Then we consider

$$
I\left(\left\{t_{j}\right\}, S_{2}+S_{1}, a, \nu\right)(x, y)=\left(\frac{E}{t_{1}}\right)^{1 / 2}\left(\frac{E}{t_{2}}\right)^{1 / 2} \int_{\mathrm{R}} e^{-i_{\nu}\left(S_{1}\left(t_{1}, x, z\right)+S_{2}\left(t_{2}, z, y\right)\right)} a(x, z, y) d z,
$$

here $a(x, z, y) \in \mathscr{B}\left(\mathbb{R}_{x} \times \mathbb{R}_{z} \times \mathbb{R}_{y}\right)$. We employ the notations $D\left(S_{2}+S_{1} ; x, y\right)$ of (2.34) in $\S 2$ and denote $\left(t_{1} t_{2}\right) /\left(t_{1}+t_{2}\right)$ by $\tau$. Applying the stationary phase method [1], we easily obtain

Lemma 3.5. Assume that $8\left(t_{1}+t_{2}\right)^{2} \kappa_{2}^{2}<1$. Then

$$
\begin{aligned}
& \left(\frac{E}{t_{1}}\right)^{1 / 2}\left(\frac{E}{t_{2}}\right)^{1 / 2} \int_{\mathrm{R}} e^{-i_{\nu}\left(S_{1}\left(t_{1}, x, z\right)+S_{2}\left(t_{2}, z, y\right)\right)} a(x, z, y) d z \\
& =\left(\frac{E}{t_{1}+t_{2}}\right)^{1 / 2} e^{-i_{\nu} S_{2,1}^{\#}} D\left(S_{2}+S_{1} ; x, y\right)^{-1 / 2} b(x, y),
\end{aligned}
$$

where $b(x, y)$ is of the following form:

$$
\begin{aligned}
b(x, y)= & \left(a\left(x, z^{*}, y\right)+\left(\frac{\tau}{i \nu}\right) D\left(S_{2}+S_{1} ; x, y\right)^{-1}\left\{\left.\frac{1}{2} \Delta_{z} a(x, z, y)\right|_{z=z^{*}}\right.\right. \\
& \left.\left.+\tau D\left(S_{2}+S_{1} ; x, y\right)^{-1} r_{1}(x, y)\right\}+\left(\frac{\tau}{i \nu}\right)^{2} D\left(S_{2}+S_{1} ; x, y\right)^{-2} r_{2}(x, y)\right),
\end{aligned}
$$


where $\Delta_{k}$ is the laplacian with respect to $z$. For each $m \geqq 0$ there exist $K(m)$ and $C_{m}$ such that for any $\alpha$ and $\beta$ with $|\alpha|,|\beta| \leqq m$

$$
\left|\partial_{x}^{\alpha} \partial_{y}^{\beta} r_{1}(x, y)\right|+\left|\partial_{x}^{\alpha} \partial_{y}^{\beta} r_{2}(x, y)\right| \leqq C_{m} \max \sup \left|\partial_{x}^{\alpha^{\prime}} \partial_{y}^{\beta^{\prime}} \partial_{z}^{\gamma^{\prime}} a(x, z, y)\right|,
$$

where $\max$ is taken for such $\alpha^{\prime}, \beta^{\prime}$ and $\gamma^{\prime}$ as $\alpha^{\prime} \leqq \alpha, \beta^{\prime} \leqq \beta, \gamma^{\prime} \leqq K(m)$. $K(m)$ can be chosen as $2 m+4+2$.

Proof. We have only to apply stationary phase method (cf. Theorem 4.1 of [1]).

Let $S_{j}\left(t_{j}, x_{j}, x_{j-1}\right), j=1,2, \cdots, L$, be the phase functions as in Lemma 3.1. We employ the notation $S_{m, k+1}^{\#}\left(x_{m}, x_{k}\right)$ of (2.35) in $\S 2$ if $m>k$.

Lemma 3.6. There exists a positive constant $\delta_{2}=\delta_{2}\left(\left\{\kappa_{m}\right\}\right)$ such that if $T_{k}=t_{k}+t_{k+1}+\cdots+t_{1}<\delta_{2}$, then

$$
\begin{aligned}
\left(\frac{E}{t_{k}}\right)^{1 / 2} & e^{-i_{\nu} S_{k}\left(t_{k}, x_{k}, x_{k-1}\right)}=\left(\frac{E}{T_{k}}\right)^{1 / 2}\left(\frac{-E}{T_{k-1}}\right)^{1 / 2} \\
& \times \tilde{\int}_{\mathrm{R}} e^{-i_{\nu}\left(s_{k, 1}^{\#}\left(x_{k}, y_{k-1}\right)-s_{k-1,1}^{\#}\left(x_{k-1}, y_{k-1}\right)\right)} b_{k}\left(x_{k}, y_{k-1}, x_{k-1}\right) d y_{k-1} .
\end{aligned}
$$

Here the function $b_{k}\left(x_{k}, y_{k-1}, x_{k-1}\right)$ satisfies the following estimate: For any $\alpha, \beta$ and $\gamma$, there is a constant $C_{\alpha \beta \gamma}$ such that

$$
\left|\partial_{x_{k}}^{\alpha} \partial_{y_{k-1}}^{\beta} \partial_{x_{k-1}}^{r} b\left(x_{k}, y_{k-1}, x_{k-1}\right)\right| \leqq C_{\alpha \beta r} .
$$

Proof. Let $\delta_{2}$ be so small that $8 \delta_{2}^{2} \kappa_{2}<1,8 \delta_{2}^{2} \kappa_{2}^{\#}<1, \delta_{2}<\delta_{1}\left(\left\{\kappa_{m}\right\}\right)$ and $\delta_{2}<\delta_{1}\left(\left\{\kappa_{2}^{*}\right\}\right)$. Assume $T_{k}<\delta_{2}$. Then $8\left(T_{k-1}+t_{k}\right)^{2} \kappa_{2}<1$ and $8\left(T_{k-1}+t_{k}\right)^{2} \kappa_{2}^{\#}<1$. So we apply Lemma 3.5 to the kernel function of the operator

$$
\mathrm{Op}\left(t_{k}, S_{k}, 1, \nu\right) \mathrm{Op}\left(T_{k-1}, S_{k-1,1}^{\#}, 1, \nu\right) .
$$

We obtain

$$
\mathrm{Op}\left(t_{k}, S_{k}, 1, \nu\right) \mathrm{Op}\left(T_{k-1}, S_{k-1,1}^{\#}, 1, \nu\right)=\mathrm{Op}\left(T_{k}, S_{k, 1}^{\sharp}, 1+\tau_{k} p_{k}, \nu\right),
$$

where $\tau_{k}=t_{k} T_{k-1}\left(t_{k}+T_{k-1}\right)^{-1}$ and $p_{k}=p_{k}\left(x_{k}, x_{0}\right) \in \mathscr{B}(\mathbb{R} \times \mathbb{R})$. Since $T_{k}<$ $\delta_{1}\left(\left\{\kappa_{m}^{\sharp}\right\}\right)$, we can apply Lemma 3.4 to $\operatorname{Op}\left(T_{k-1}, S_{k-1,1}^{\#}, 1, \nu\right)$. Therefore, we have

$$
\begin{aligned}
\mathrm{Op}\left(t_{k}, S_{k}, 1, \nu\right) & =\operatorname{Op}\left(T_{k}, S_{k, 1}^{\#}, 1+\tau_{k} p_{k}, \nu\right) \operatorname{Op}\left(T_{k-1}, S_{k-1,1}^{\#}, 1, \nu\right)^{-1} \\
& =\operatorname{Op}\left(T_{k}, S_{k, 1}^{\#}, 1+\tau_{k} p_{k}, \nu\right) \operatorname{Op}\left(T_{k-1}, S_{k-1,1}^{\#}, 1+T_{k-1} q_{k}, \nu\right)^{*}
\end{aligned}
$$

This means that 


$$
\begin{aligned}
& \left(\frac{E}{t_{k}}\right)^{1 / 2} e^{-i \nu S_{k}\left(t_{k}, x_{k}, x_{k-1}\right)}=\left(\frac{E}{T_{k}}\right)^{1 / 2}\left(\frac{-E}{T_{k-1}}\right)^{1 / 2} \\
& \quad \times \tilde{\int}_{\mathrm{R}} e^{-i_{\nu}\left(S_{k, 1}^{\sharp}\left(x_{k}, y_{k-1}\right)-S_{k-1,1}^{\#}\left(x_{k-1}, y_{k-1}\right)\right)} b_{k}\left(x_{k}, y_{k-1}, x_{k-1}\right) d y_{k-1}
\end{aligned}
$$

with

$$
b_{k}\left(x_{k}, y_{k-1}, x_{k-1}\right)=\left(1+\tau_{k} p_{k}\left(x_{k}, y_{k-1}\right)\right)\left(1+T_{k-1} q_{k}\left(x_{k-1}, y_{k-1}\right)\right) \text {. }
$$

Lemma 3.6 is proved.

We can now prove Lemma 3.1. The proof is a modification of the discussion in [6], [7], [8] and [4]. Let $\delta$ be so small that $\delta<\delta_{1}\left(\left\{\kappa_{m}\right\}\right)$ and $\delta<\delta_{2}\left(\left\{\kappa_{m}\right\}\right)$ and $8 \delta^{2} \kappa_{2}<1$. Assume that $T_{L}<\delta$. Using the function $I\left(\left\{t_{j}\right\}, S, a, \nu\right)\left(x_{L}, x_{0}\right)$, we define integral transform

$$
\mathrm{Op}\left(\left\{t_{j}\right\}, S, a, \nu\right) f\left(x_{L}\right)=\tilde{\int}_{\mathrm{R}} I\left(\left\{t_{j}\right\}, S, a, \nu\right)\left(x_{L}, x_{0}\right) f\left(x_{0}\right) d x_{0} .
$$

Since $8 T_{L}^{2} \kappa_{2}<1, \operatorname{Op}\left(\left\{t_{j}\right\}, S, a, \nu\right)$ is a bounded operator on $L^{2}(\mathbb{R})$. (cf. [1]).

Since $T_{L}<\delta_{2}\left(\left\{\kappa_{m}\right\}\right)$, we can apply Lemma 3.6 to $e^{-i \nu S_{k}\left(t, x_{k}, x_{k-1}\right)}$ for any $k=2,3, \cdots, L$. Thus

$$
\begin{aligned}
\mathrm{Op}\left(\left\{t_{j}\right\},\right. & S, a, \nu) f\left(x_{L}\right)=\prod_{k=1}^{L}\left(\frac{E}{t_{k}}\right)^{1 / 2} \int_{\mathrm{R}^{L}} e^{-i \nu\left(\Sigma s_{j}\left(t_{j}, x_{j}, x_{j-1}\right)\right)} a\left(x_{L}, \cdots, x_{0}\right) f\left(x_{0}\right) \prod_{j=0}^{L-1} d x_{j} \\
= & \left(\frac{E}{t_{1}}\right)^{1 / 2} \prod_{j=2}^{L}\left(\frac{E}{T_{j}}\right)^{1 / 2}\left(\frac{-E}{T_{j-1}}\right)^{1 / 2} \int_{\mathrm{R}^{2(L-1)+1}} e^{-i_{\nu} \Phi\left(x_{L}, y_{L-1}, x_{L-1}, \cdots, y_{1}, x_{1}, x_{0}\right)} \\
& \times a\left(x_{L}, \cdots, x_{0}\right) \prod_{j=2}^{L} b_{j}\left(x_{j}, y_{j-1}, x_{j-1}\right) f\left(x_{0}\right) d x_{0} \prod_{k=1}^{L-1} d x_{j} d y_{j}
\end{aligned}
$$

where the phase function $\Phi$ equals

$$
\begin{aligned}
& \Phi\left(x_{L}, y_{L-1}, x_{L-1}, \cdots, y_{1}, x_{1}, x_{0}\right) \\
& =\sum_{j=2}^{L}\left\{S_{j, 1}^{\#}\left(x_{j}, y_{j-1}\right)-S_{j-1,1}^{\#}\left(x_{j-1}, y_{j-1}\right)\right\}+S_{1}\left(t_{1}, x_{1}, x_{0}\right) \\
& =S_{L, 1}^{\#}\left(x_{L}, y_{L-1}\right)+\sum_{j=2}^{L-1}\left\{S_{j, 1}^{\#}\left(x_{j}, y_{j-1}\right)-S_{j, 1}^{\#}\left(x_{j}, y_{j}\right)\right\} \\
& \quad+S_{1}\left(t_{1}, x_{1}, x_{0}\right)-S_{1}\left(t_{1}, x_{1}, y_{1}\right) .
\end{aligned}
$$

We next employ Kuranishi's technique. We rewrite

$$
S_{j, 1}^{\sharp}\left(x_{j}, y_{j-1}\right)-S_{j, 1}^{\#}\left(x_{j}, y_{j}\right)=\frac{y_{j-1}-y_{j}}{T_{j}} \xi_{j},
$$


where

$$
\begin{aligned}
\xi_{j} & =T_{j} \int_{0}^{1} \partial_{y} S_{j, 1}^{\sharp}\left(x_{j}, s y_{j-1}+(1-s) y_{j}\right) d s \\
& =-x_{j}+\frac{1}{2}\left(y_{j-1}+y_{j}\right)+T_{j} \int_{0}^{1} \partial_{y} \omega_{j, 1}^{\sharp}\left(x_{j}, s y_{j-1}+(1-s) y_{j}\right) d s .
\end{aligned}
$$

Therefore, the jacobian of the correspondence $x_{j} \rightarrow \xi_{j}$ is

$$
\frac{\partial \xi_{j}}{\partial x_{j}}=-I+T_{j} p\left(y_{j}, x_{j}, y_{j-1}\right)
$$

where

$$
p_{j}\left(y_{j}, x_{j}, y_{j-1}\right)=\int_{0}^{1} \partial_{x_{j}} \partial_{y} \omega_{j, 1}^{\#}\left(x_{j}, s y_{j-1}+(1-s) y_{j}\right) d s
$$

satisfies the estimate: For any $\alpha, \beta$ and $\gamma$, with $|\alpha|,|\beta|$ and $|\gamma|<m$,

$$
\left|\partial_{y_{j}}^{\alpha} \partial_{x_{j}}^{\beta} \partial_{y_{j-1}}^{r} p\left(y_{j}, x_{j}, y_{j-1}\right)\right| \leqq \kappa_{m+2}^{\#} .
$$

Since $8 T_{L}^{2} \kappa_{2}^{\#}<1$, we have $\left|\partial \xi_{j} / \partial x_{j}\right|<2^{-1}$. The correspondence $\mathbb{R} \in x_{j} \rightarrow$ $\xi_{j} \in \mathbb{R}$ is one to one and onto. We may consider $x_{j}$ as a function $x_{j}\left(y_{j}, \xi_{j}, y_{j-1}\right)$.

This diffeomorphism has the following property: Let $f\left(y_{j}, x_{j}, y_{j-1}\right)$ be an arbitrary function in $\mathscr{B}(\mathbb{R} \times \mathbb{R} \times \mathbb{R})$. Then for any multi-indices $\alpha, \beta$ and $\gamma$ there exists a positive constant $C_{\alpha \beta r}$ such that

$$
\left|\partial_{y_{j}}^{\alpha} \partial_{\xi_{j}}^{\beta} \partial_{y_{j-1}}^{r} f\left(y_{j}, x_{j}\left(y_{j}, \xi_{j}, y_{j-1}\right), y_{j-1}\right)\right|<C_{\alpha \beta \gamma} \max \sup \left|\partial_{y_{j}}^{\alpha^{\prime}} \partial_{x_{j}}^{\beta^{\prime}} \partial_{y_{j-1}}^{\gamma^{\prime}} f\left(y_{j}, x_{j}, y_{j-1}\right)\right|,
$$

where maximum is taken with respect to those multi-indices $\alpha^{\prime} \leqq \alpha, \beta^{\prime} \leqq \beta$ and $\gamma^{\prime} \leqq \gamma$.

Let $\eta_{j}=T_{j}^{-1} \xi_{j}\left(y_{j}, x_{j}, y_{j-1}\right)$. Then for any $\alpha, \beta$ and $\gamma$ there exists a constant $C_{\alpha \beta r}$ independent of $\left\{t_{j}\right\}_{j}$ such that

$$
\begin{aligned}
& \left|\partial_{y_{j}}^{\alpha} \partial_{\eta_{j}}^{\beta} \partial_{y_{j-1}}^{r} f\left(y_{j}, x_{j}\left(y_{j}, T_{j} \eta_{j}, y_{j-1}\right), y_{j-1}\right)\right| \\
& \quad \leqq C_{\alpha \beta \gamma} T_{j}^{|\beta|} \max \sup _{\mathbf{R} \times \mathbf{R} \times \mathbf{R}}\left|\partial_{y_{j}}^{\alpha^{\prime}} \partial_{x_{j}}^{\beta^{\prime}} \partial_{y_{j-1}}^{\gamma^{\prime}} f\left(y_{j}, x_{j}, y_{j-1}\right)\right|,
\end{aligned}
$$

where max is taken with respect to multi-indices with $\alpha^{\prime} \leqq \alpha, \beta^{\prime} \leqq \beta$ and $\gamma^{\prime} \leqq \gamma$

Similarly, we make change of variables from $x_{1}$ to $\eta_{1}$.

$$
S_{1}\left(t_{1}, x_{1}, x_{0}\right)-S_{1}\left(t_{1}, x_{1}, y_{1}\right)=\eta_{1}\left(x_{0}-y_{1}\right),
$$


here

$$
\eta_{1}=\frac{-1}{t_{1}}\left(\left(x_{1}-\frac{1}{2}\left(x_{0}+y_{1}\right)\right)+\int_{0}^{1} \partial_{x_{0}} \omega_{1}\left(t_{1}, x_{1}, s x_{0}+(1-s) y_{1}\right) d s .\right.
$$

After these change of variables the phase function becomes

$$
\Phi=S_{L, 1}^{\#}\left(x_{L}, y_{L-1}\right)+\sum_{j=2}^{L-1} \eta_{j}\left(y_{j-1}-y_{j}\right)+\eta_{1}\left(x_{0}-y_{1}\right),
$$

where $y_{L}=x_{L}$ and $y_{0}=x_{0}$. Therefore we have

$$
\mathrm{Op}\left(\left\{t_{j}\right\}, S, a, \nu\right) f\left(y_{L}\right)=\left(\frac{E}{T_{L}}\right)^{1 / 2} \int_{\mathrm{R}} e^{-i \nu S_{L, 1}^{\#}\left(y_{L}, y_{L-1}\right)} J(f)\left(y_{L}, y_{L-1}\right) d y_{L-1},
$$

where

$$
\begin{aligned}
J(f)\left(y_{L}, y_{L-1}\right) & =\left(\frac{\nu}{2 \pi}\right)^{L-1} \int_{\mathrm{R}^{2}(L-1)} \exp \left\{-i \nu\left(\sum_{j=1}^{L-1}\left(y_{j}-y_{j-1}\right) \eta_{j}\right)\right\} \\
& \times a_{1}\left(y_{L}, y_{L-1}, \eta_{L-1} \cdots, y_{1}, \eta_{1}, y_{0}\right) f\left(y_{0}\right) \prod_{j=1}^{L-1} d y_{j-1} d \eta_{j}
\end{aligned}
$$

with

$$
\begin{aligned}
& a_{1}\left(y_{L}, y_{L-1}, \eta_{L-1}, \cdots, y_{1}, \eta_{1}, y_{0}\right) \\
& \quad=a\left(x_{L}, \cdots, x_{0}\right) \prod_{k=2}^{L} b_{k}\left(x_{k}, y_{k}, x_{k-1}\right) \prod_{j=1}^{L-1}\left|\frac{\partial x_{j}}{\partial \xi_{j}}\right| .
\end{aligned}
$$

We consider $y_{L}$ as a parameter and apply Lemma 3.2 to $J(f)\left(y_{L}, y_{L-1}\right)$. Then we obtain

$$
J(f)\left(y_{L}, y_{L-1}\right)=\left(\frac{\nu}{2 \pi}\right) \int_{\mathrm{R}^{2}} e^{-i_{\nu}\left(y_{L-1}-y_{0}\right) \eta} U\left(a_{1}\right)\left(y_{L}, y_{L-1}, \eta, y_{0}\right) f\left(y_{0}\right) d y_{0} d \eta
$$

For any multi-indices $\alpha_{L}, \alpha_{L-1}, \alpha_{0}$ and $\beta$ with $\left|\alpha_{L}\right|,\left|\alpha_{L-1}\right|,\left|\alpha_{0}\right|,|\beta| \leqq m_{1}$, we have the following estimate:

$$
\begin{aligned}
& \left|\partial_{y_{L}}^{\alpha_{L}} \partial_{y_{L-1}}^{\alpha_{L-1}} \partial_{\eta}^{\beta} \partial_{y_{0}}^{\alpha_{0}} U\left(a_{1}\right)\left(y_{L}, y_{L-1}, \eta, y_{0}\right)\right| \\
& \quad \leqq C_{1} C_{2}^{L-1} \max \sup \left|\partial_{y_{L}}^{\alpha_{L}} \partial_{y_{L-1}}^{\alpha_{L-1}} \prod_{k=2}^{L-1}\left(\partial_{y_{k-1}}^{\alpha_{k-1}^{\prime}} \partial_{\eta_{k}}^{\beta_{k}^{\prime}}\right) \partial_{\eta_{1}}^{\beta_{1}^{\prime}} \partial_{y_{0}}^{\alpha_{0}^{\prime}} a_{1}\left(y_{L}, y_{L-1}, \eta_{L-1}, \cdots, y_{0}\right)\right|
\end{aligned}
$$

Here $C_{1}$ and $C_{2}$ are positive constants depending on $m_{1}$, $\max$ is taken with respect to multi-indices with $\left|\alpha_{L-1}\right|,\left|\alpha_{k}^{\prime}\right|,\left|\beta_{k}^{\prime}\right| \leqq K\left(m_{1}\right) \leqq 2 m_{1}+3$ and sup is taken with respect to $y_{j} \in \mathbb{R}, \eta_{j} \in \mathbb{R}, j=1, \cdots, L-1$. Since relationship $a_{1}\left(x_{L}, \cdots, x_{0}\right)$ with $a\left(x_{L}, \cdots, x_{0}\right)$ is given by (3.2), we have 


$$
\begin{aligned}
& \left|\partial_{y_{L}}^{\alpha_{L}} \partial_{y_{L-1}}^{\alpha_{L-1}} \partial_{\eta}^{\beta} \partial_{y_{0}}^{\alpha_{0}} U\left(a_{1}\right)\left(y_{L}, y_{L-1}, \eta, y_{0}\right)\right| \\
& \quad \leqq C_{1} C_{3}^{L-1} \max \sup _{x_{L-1} \cdots x_{1}}\left|\partial_{x_{L}}^{\alpha_{L}^{\prime \prime}} \partial_{x_{L-1}}^{\alpha_{L}} \cdots \partial_{x_{0}}^{\alpha_{0}^{\prime \prime}} a\left(x_{L}, x_{L-1}, \cdots, x_{0}\right)\right|
\end{aligned}
$$

where $C_{3}$ is another constant and max is taken with respect to multiindices satisfying $\left|\alpha_{L}^{\prime \prime}\right|,\left|\alpha_{L-1}^{\prime \prime}\right|, \cdots,\left|\alpha_{0}^{\prime \prime}\right| \leqq 2 m_{1}+3$.

We replace $J(f)\left(y_{L}, y_{L-1}\right)$ in (3.1) by the right hand side of (3.3). Then we have the expression

$$
\begin{aligned}
& \mathrm{Op}\left(\left\{t_{j}\right\}, S, a, \nu\right) f\left(x_{L}\right)=\left(\frac{E}{T_{L}}\right)^{1 / 2}\left(\frac{\nu}{2 \pi}\right) \\
& \quad \times \int_{\mathrm{R}^{3}} e^{-i_{\nu}\left(S_{L, 1}^{\#}\left(x_{L}, y_{L-1}\right)+\left(y_{L-1}-y_{0}\right) \eta\right)} U\left(a_{1}\right)\left(x_{L}, y_{L-1}, \eta, y_{0}\right) f\left(y_{0}\right) d y_{0} d \eta d y_{L-1} .
\end{aligned}
$$

Using stationary phase method with respect to $y_{L-1}$ and $\eta$, we can find $b\left(x_{L}, x_{0}\right)$ such that

$$
\left.\mathrm{Op}\left(\left\{t_{j}\right\}\right), S, a, \nu\right) f\left(x_{L}\right)=\left(\frac{E}{T_{L}}\right)^{1 / 2} \int_{\mathbf{R}} e^{-i \nu S_{L, 1}^{\sharp}\left(x_{L}, x_{0}\right)} b\left(x_{L}, x_{0}\right) f\left(x_{0}\right) d x_{0} .
$$

This means that

$$
I\left(\left\{t_{j}\right\}, S, a, \nu\right)\left(x_{L}, x_{0}\right)=\left(\frac{E}{T_{L}}\right)^{1 / 2} e^{-i \nu S_{L, 1}^{\#}\left(x_{L}, x_{0}\right)} b\left(x_{L}, x_{0}\right) .
$$

Here $b$ satisfies the following estimate: For any $m \geqq 0$ there exists a positive constant $C(m)$ such that if $\left|\alpha_{L}\right|,\left|\alpha_{0}\right| \leqq m$

$$
\left|\partial_{x_{L}}^{\alpha_{L}} \partial_{x_{0}}^{\alpha_{0}} b\left(x_{L}, x_{0}\right)\right| \leqq C(m) \max _{y_{L-1}, \eta}\left|\partial_{x_{L}}^{\alpha_{L}^{\prime}} \partial_{y_{L-1}}^{\alpha_{L}^{\prime}} \partial_{\eta}^{\beta^{\prime}} \partial_{y_{0}}^{\alpha_{0}} U\left(a_{1}\right)\left(x_{L}, y_{L-1}, \eta, y_{0}\right)\right|
$$

where $\max$ is taken with respect to multi-indices satisfying $\alpha_{L}^{\prime} \leqq \alpha_{L}$, $\alpha_{0}^{\prime} \leqq \alpha_{0}$ and $\left|\alpha_{L-1}^{\prime}\right|,\left|\beta^{\prime}\right|<2 m+10$. Combining (3.7) with (3.4), we have

$$
\left|\partial_{x_{L}}^{\alpha_{L}} \partial_{x_{0}}^{\alpha_{0}} b\left(x_{L}, x_{0}\right)\right| \leqq C_{4} C_{5}^{L-1} \max \sup _{x_{L-1}, \cdots, x_{1}}\left|\partial_{x_{L}}^{\alpha_{L}^{\prime \prime}} \partial_{x_{L-1}}^{\alpha_{L}^{\prime \prime}} \cdots \partial_{x_{0}}^{\alpha_{0}^{\prime \prime}} a\left(x_{L}, x_{L-1}, \cdots, x_{0}\right)\right|
$$

where $C_{4}, C_{5}$ are positive constants depending on $m$ and $\max$ is taken with respect to multi-indices satisfying $\left|\alpha_{L}^{\prime \prime}\right|,\left|\alpha_{L-1}^{\prime \prime}\right|, \cdots,\left|\alpha_{0}^{\prime \prime}\right| \leqq 2(2 m+10)$ $+3=4 m+23$. This together with (3.6) above proves Lemma 3.1 with $K(m)=4 m+23$.

\section{§4. Proof of Theorem 1}

For any $k>j$, we denote $t_{k}+\cdots+t_{j}$ by $T(k, j)$. Let $\delta$ be as in Lemma 3.1. We have to treat the oscillatory integral 


$$
\begin{aligned}
& I\left(\left\{t_{j}\right\}, S, a, \nu\right)\left(x_{L}, x_{0}\right) \\
& \quad=\prod_{j=1}^{L}\left(\frac{E}{t_{j}}\right)^{1 / 2} \int_{R^{(L-1)}} \exp \left\{-i \nu \sum_{j=1}^{L} S_{j}\left(t_{j}, x_{j}, x_{j-1}\right)\right\} a\left(x_{L}, \cdots, x_{0}\right) \prod_{j=1}^{L-1} d x_{j},
\end{aligned}
$$

when $T_{L}<\delta$.

First we perform integration over $x_{1}$ space. Using stationary phase method, we have

$$
\begin{aligned}
& \left(\frac{E}{t_{2}}\right)^{1 / 2}\left(\frac{E}{t_{1}}\right)^{1 / 2} \int_{\mathrm{R}} e^{-i_{\nu}\left(S_{2}\left(t_{2}, x_{2}, x_{1}\right)+S_{1}\left(t_{1}, x_{1}, x_{0}\right)\right)} a\left(x_{L}, \cdots, x_{0}\right) d x_{1} \\
& \quad=\left(\frac{E}{T(2,1)}\right)^{1 / 2} e^{-i_{\nu} S_{2,1}^{\#}\left(x_{2}, x_{0}\right)}\left(\left(S_{1} a\right)\left(x_{L}, \cdots, x_{2}, x_{0}\right)+\left(R_{1} a\right)\left(x_{L}, \cdots, x_{2}, x_{0}\right)\right) .
\end{aligned}
$$

The amplitude of the main term of the right hand side equals

$$
\left(S_{1} a\right)\left(x_{L}, \cdots, x_{2}, x_{0}\right)=a\left(x_{L}, \cdots, \widehat{x_{2}, x_{0}}\right) D\left(S_{2}+S_{1} ; x_{2}, x_{0}\right)^{-1 / 2},
$$

and $R_{1} a\left(x_{L}, \cdots, x_{2}, x_{0}\right)$ is the remainder term.

Similarly, integrating $S_{1} a$ over $x_{2}$ space and applying the stationary phase method, we obtain

$$
\begin{aligned}
& \left(\frac{E}{t_{3}}\right)^{1 / 2}\left(\frac{E}{T(2,1)}\right)^{1 / 2} \int_{\mathrm{R}}^{\tilde{e}} e^{-i_{\nu}\left(S_{3}\left(t_{3}, x_{3}, x_{2}\right)+S_{2,1}^{\#}\left(x_{2}, x_{0}\right)\right)} S_{1} a\left(x_{L}, \cdots, x_{2}, x_{0}\right) d x_{2} \\
& \quad=\left(\frac{E}{T(3,1)}\right)^{1 / 2} e^{-i \nu_{3,1}^{\#}\left(x_{3}, x_{0}\right)}\left(S_{2} S_{1} a\left(x_{L}, \cdots, x_{3}, x_{0}\right)+R_{2} S_{1} a\left(x_{L}, \cdots, x_{3}, x_{0}\right)\right),
\end{aligned}
$$

where $S_{2} S_{1} a$ is the main term and $R_{2} S_{1} a$ is the remainder term. We have

$$
S_{2} S_{1} a\left(x_{L}, \cdots, x_{0}\right)=D\left(S_{3}+S_{2,1}^{\#} ; x_{3}, x_{0}\right)^{-1 / 2}\left(S_{1} a\right)\left(x_{L}, \cdots, x_{3}, x_{2}^{*}, x_{1}\right),
$$

where $x_{2}^{*}$ is the critical point of $S_{3}+S_{2,1}^{\#}$ with respect to $x_{2}$.

When we integrate the term including $S_{2} S_{1} a\left(x_{L}, \cdots, x_{3}, x_{0}\right)$ over $x_{3}$ space, we use the stationary phase method:

$$
\begin{aligned}
& \left(\frac{E}{t_{4}}\right)^{1 / 2}\left(\frac{E}{T(3,1)}\right)^{1 / 2} \int_{\mathrm{R}}^{\tilde{2}} e^{-i_{\nu}\left(S_{4}\left(t_{4}, x_{4}, x_{3}\right)+S_{3,1}^{\sharp}\left(x_{3}, x_{0}\right)\right)} S_{2} S_{1} a\left(x_{L}, \cdots, x_{3}, x_{0}\right) d x_{3} \\
& \quad=\left(\frac{E}{T(4,1)}\right)^{1 / 2} e^{-i \nu S_{4,1}^{\sharp}\left(x_{4}, x_{0}\right)}\left(S_{3} S_{2} S_{1} a\left(x_{L}, \cdots, x_{4}, x_{0}\right)+R_{3} S_{2} S_{1} a\left(x_{L}, \cdots, x_{4}, x_{0}\right)\right) .
\end{aligned}
$$

$S_{3} S_{2} S_{1} a$ is the main term and $R_{3} S_{2} S_{1} a$ is the remainder.

Repeating this process $L-1$ times, finally we obtain, among other terms, 


$$
\left(\frac{E}{T_{L}}\right)^{1 / 2} e^{-i \nu S_{L, 1}^{\#}\left(x_{L}, x_{0}\right)} S_{L-1} S_{L-2} \cdots S_{1}(a)\left(x_{L}, x_{0}\right)
$$

Since we have

$$
S_{L-1} S_{L-2} \cdots S_{1} a\left(x_{L}, x_{0}\right)=\left.\prod_{k=2}^{L} D\left(S_{k}+S_{k-1,1}^{\#} ; x_{k}, x_{0}\right)^{-1 / 2} a(x)\right|_{x_{L-1}^{*}, \cdots x_{1}^{*}}
$$

Proposition 2.8 yields that

$$
S_{L-1} S_{L-2} \cdots S_{1} a\left(x_{L}, x_{0}\right)=D\left(x_{L}, x_{0}\right)^{-1 / 2} a\left(\widehat{x_{L}, x_{0}}\right) .
$$

Here $D\left(x_{L}, x_{0}\right)=\left(t_{1} t_{2} \cdots t_{L} /\left(t_{1}+\cdots+t_{L}\right)\right)$ det $\operatorname{Hess}_{x^{*}}\left(S_{L}+\cdots+S_{1}\right)$ as in $\S 2$. Therefore,

$$
\begin{aligned}
& \left(\frac{E}{T_{L}}\right)^{1 / 2} e^{-i \nu S_{L, 1}^{\sharp}\left(x_{L}, x_{0}\right)} S_{L-1} S_{L-2} \cdots S_{1}(a)\left(x_{L}, x_{0}\right) \\
& =\left(\frac{E}{T_{L}}\right)^{1 / 2} e^{-i \nu S_{L, 1}^{\sharp}\left(x_{L}, x_{0}\right)} D\left(x_{L}, x_{0}\right)^{-1 / 2} a\left(\widetilde{x_{L}, x_{0}}\right) .
\end{aligned}
$$

This is nothing but the main term of Theorem 1 . The remainder term consists of others.

Now we treat the remainder term. Since $\left(R_{1} a\right)\left(x_{L}, \cdots, x_{2}, x_{0}\right)$ has complicated structure as a function of $x_{2}$, we postpone integration over $x_{2}$ space of the term including $\left(R_{1} a\right)\left(x_{L}, \cdots, x_{2}, x_{0}\right)$ until later stage of the proof. We do perform integration over $x_{3}$ space beforehand, because the structure of $R_{1} a\left(x_{L}, \cdots, x_{3}, x_{2}, x_{0}\right)$ as a function of $x_{3}$ is much simpler. The stationary phase method gives

$$
\begin{aligned}
\left(\frac{E}{t_{4}}\right)^{1 / 2} & \left(\frac{E}{t_{3}}\right)^{1 / 2}\left(\frac{E}{T(2,1)}\right)^{1 / 2} \int_{\mathrm{R}} e^{-i_{\nu}\left(S_{4}\left(t_{4}, x_{4}, x_{3}\right)+S_{3}\left(t_{3}, x_{3}, x_{2}\right)+S_{2,1}^{\#}\left(x_{2}, x_{0}\right)\right)} R_{1} a\left(x_{L}, \cdots, x_{2}, x_{0}\right) d x_{3} \\
= & \left(\frac{E}{T(4,3)}\right)^{1 / 2}\left(\frac{E}{T(2,1)}\right)^{1 / 2} e^{-i_{\nu}\left(S_{4,3}^{\sharp}\left(x_{4}, x_{2}\right)+S_{2,1}^{\#}\left(x_{2}, x_{0}\right)\right)} \\
& \times\left(S_{3} R_{1} a\left(x_{L}, \cdots, x_{4}, x_{2}, x_{0}\right)+R_{3} R_{1} a\left(x_{L}, \cdots, x_{4}, x_{2}, x_{0}\right)\right) .
\end{aligned}
$$

Again $S_{3} R_{1} a\left(x_{L}, \cdots, x_{4}, x_{2}, x_{0}\right)=\left(R_{1} a\right)\left(x_{L}, \cdots, \overrightarrow{x_{4}, x_{2}}, x_{0}\right) D\left(S_{4}+S_{3} ; x_{4}, x_{2}\right)^{-1 / 2}$ is the main term and $R_{3} R_{1} a\left(x_{L}, \cdots, x_{4}, x_{2}, x_{0}\right)$ is the remainder.

We skip integration over $x_{3}$ space of the term including $R_{2} S_{1} a\left(x_{L}\right.$, $\left.\cdots, x_{3}, x_{1}\right)$, because this is complicated as a function of $x_{3}$. By virtue of the stationary phase method, 


$$
\begin{aligned}
&\left(\frac{E}{t_{5}}\right)^{1 / 2}\left(\frac{E}{t_{4}}\right)^{1 / 2}\left(\frac{E}{T(3,1)}\right)^{1 / 2} e^{-i \nu S_{3,1}^{\#}\left(x_{3}, x_{0}\right)} \\
& \quad \times \int_{\mathrm{R}} e^{-i \nu\left(S_{5}\left(t_{5}, x_{5}, x_{4}\right)+S_{4}\left(t_{4}, x_{4}, x_{3}\right)\right)} R_{2} S_{1} a\left(x_{L}, \cdots, x_{4}, x_{3}, x_{0}\right) d x_{4} \\
&=\left(\frac{E}{T(5,4)}\right)^{1 / 2}\left(\frac{E}{T(3,1)}\right)^{1 / 2} e^{-i \nu\left(S_{5,3}^{\#}\left(x_{5}, x_{3}\right)+S_{3,1}^{\#}\left(x_{3}, x_{0}\right)\right)} \\
& \times\left(S_{4} R_{2} S_{1} a\left(x_{L}, \cdots, x_{5}, x_{3}, x_{0}\right)+R_{4} R_{2} S_{1} a\left(x_{L}, \cdots, x_{5}, x_{3}, x_{0}\right)\right) .
\end{aligned}
$$

Here

$$
S_{4} R_{2} S_{1} a\left(x_{L}, \cdots, x_{5}, x_{3}, x_{0}\right)=\left(R_{2} S_{1} a\right)\left(x_{L}, \cdots, \overline{x_{5}, x_{3}}, x_{0}\right) D\left(S_{5}+S_{4} ; x_{5}, x_{3}\right)^{-1 / 2}
$$

is the main term and $R_{4} R_{2} S_{1} a$ is the remainder.

Similarly, we perform integration over $x_{4}$ space of the term including $S_{3} R_{1} a$. But we skip integration over $x_{4}$ space of the term including $R_{3} R_{1} a$.

We continue this process; the rule is that if $R_{k}$ appears we skip integration over $x_{k+1}$ space. Then, finally, we get the expression

$$
I\left(\left\{t_{j}\right\}, S, a, \nu\right)\left(x_{L}, x_{0}\right)=A_{0}\left(x_{L}, x_{0}\right)+\sum^{\prime} A_{j_{s} j_{s-1} j_{s-2} \cdots j_{1}}\left(x_{L}, x_{0}\right) .
$$

Here the main term is

$$
\begin{aligned}
A_{0}\left(x_{L}, x_{0}\right) & =\left(\frac{E}{T_{L}}\right)^{1 / 2} e^{-i \nu S_{L, 1}^{\sharp}\left(x_{L}, x_{0}\right)} S_{L-1} S_{L-2} \cdots S_{1} a\left(x_{L}, x_{0}\right) \\
& =\left(\frac{E}{T_{L}}\right)^{1 / 2} e^{-i \nu S_{L, 1}^{\sharp}\left(x_{L}, x_{0}\right)} D\left(x_{L}, x_{0}\right)^{-1 / 2} a\left(\widetilde{x_{L}, x_{0}}\right)
\end{aligned}
$$

$\sum^{\prime}$ stands for the summation with respect to sequence of integers $\left(j_{s}, j_{s-1}\right.$, $\cdots, j_{1}$ ) with the property

$$
\begin{aligned}
0 & =j_{0}<j_{1}-1<j_{1}<j_{2}-1<j_{2}<j_{3}-1<\cdots<j_{s-1}<j_{s}-1<j_{s} \\
& \leqq L-1<j_{s+1}=L .
\end{aligned}
$$

The summand is

$$
\begin{aligned}
A_{j_{s} j_{s-1} \cdots j_{1}}\left(x_{L}, x_{0}\right)= & \prod_{m=1}^{s}\left(\frac{E}{T\left(j_{m}, j_{m-1}+1\right)}\right)^{1 / 2} \\
& \times \int_{R^{s}} \exp \left\{-i \nu S_{j_{s} j_{s-1} \cdots j_{1}}^{\sharp}\left(x_{L}, x_{j_{s}}, \cdots, x_{j_{1}}, x_{0}\right)\right\} \\
& \times b_{j_{s} j_{s-1} \cdots j_{1}}\left(x_{L}, x_{j_{s}}, \cdots, x_{j_{1}}, x_{0}\right) \prod_{u=1}^{s} d x_{j_{u}} .
\end{aligned}
$$


The amplitude function of this is

$$
\begin{aligned}
& b_{j_{s} j_{s-1} \cdots j_{1}}\left(x_{L}, x_{j_{s}}, x_{j_{s-1}}, \cdots, x_{j_{1}}, x_{0}\right) \\
& \quad=\left(Q_{L-1} Q_{L-2} \cdots Q_{1} a\right)\left(x_{L}, x_{j_{s}}, \cdots, x_{j_{1}}, x_{0}\right),
\end{aligned}
$$

where $\quad Q_{j}=\mathrm{Id}, \quad$ if $j=j_{s}, j_{s-1}, \cdots, j_{1}$;

$=R_{j}, \quad$ if $j=j_{s}-1, j_{s-1}-1, \cdots, j_{1}-1$;

$=S_{j}, \quad$ otherwise.

The phase function is

$$
S_{j_{s} j_{s-1} \cdots j_{1}}^{\sharp}\left(x_{L}, x_{j_{s}}, \cdots, x_{j_{1}}, x_{0}\right)=\sum_{k=1}^{s+1} S_{j_{k}, j_{k-1}+1}^{\#}\left(x_{j_{k}}, x_{j_{k-1}}\right) .
$$

We can apply Lemma 3.1 to $A_{j_{s} j_{s-1} \cdots j_{1}}$ and obtain

$$
A_{j_{s} j_{s-1} \cdots j_{1}}\left(x_{L}, x_{0}\right)=\left(\frac{E}{T_{L}}\right)^{1 / 2} e^{-i \nu S_{L, 1}^{*}\left(x_{L}, x_{0}\right)} a_{j_{s} j_{s-1} \cdots j_{1}}\left(x_{L}, x_{0}\right) .
$$

For any $m \geqq 0$ Lemma 3.1 gives positive constants $C_{m}$ and $K(m)$ such that if $\left|\alpha_{L}\right|$ and $\left|\alpha_{0}\right| \leqq m$

$$
\begin{aligned}
& \left|\partial_{x_{L}}^{\alpha L} \partial_{x_{0}}^{\alpha_{0}} a_{j_{s} j_{s-1} \cdots j_{1}}\left(x_{L}, x_{0}\right)\right| \\
& \quad \leqq C_{m}^{s} \max \sup _{x_{j_{u}}}\left|\partial_{x_{L}}^{\beta_{L}} \partial_{x_{s_{s}}}^{\beta_{j_{s}}} \cdots \partial_{x_{j_{1}}}^{\beta_{j_{1}}} \partial_{x_{0}}^{\beta_{0}} b_{j_{s} j_{s-1} \cdots j_{1}}\left(x_{L}, x_{j_{s}}, \cdots, x_{j_{1}}, x_{0}\right)\right| .
\end{aligned}
$$

Here max is taken over those indices $\beta$ 's which satisfy $\beta_{L} \leqq \alpha_{L},\left|\beta_{0}\right| \leqq m$, $\left|\beta_{j_{k}}\right| \leqq K(m)$ for $k=1,2, \cdots, s$ and sup is taken with respect to $x_{j_{u}} \in \mathbb{R}$, $u=1, \cdots, s$. This implies that

$$
\begin{aligned}
& I\left(\left\{t_{\}}\right\}, S, a, \nu\right)\left(x_{L}, x_{0}\right) \\
& =\left(\frac{E}{T_{L}}\right)^{1 / 2} e^{-i \nu S_{L, 1}^{\#}\left(x_{L}, x_{0}\right)}\left(D\left(x_{L}, x_{0}\right)^{-1 / 2}\left(a\left(\widehat{x_{L}, x_{0}}\right)+r\left(x_{L}, x_{0}\right)\right)\right), \\
& \quad r\left(x_{L}, x_{0}\right)=D\left(x_{L}, x_{0}\right)^{1 / 2} \sum^{\prime} a_{j_{s} j_{s-1} \ldots j_{1}}\left(x_{L}, x_{0}\right) .
\end{aligned}
$$

Therefore, we have only to obtain estimate of $\sum^{\prime} a_{j_{s}} \cdots_{j_{1}}\left(x_{L}, x_{0}\right)$ for the proof of Theorem 1 .

In order to prove the estimate of $a_{j_{s} j_{s-1} \ldots j_{1}}\left(x_{L}, x_{0}\right)$ we can use estimate of $b_{j_{s} j_{s-1} \cdots j_{1}}$, because (4.12) holds.

LEMma 4.1. Assume (H.1) for the phase function and (H.2) for the amplitude function. Let $\delta$ be as in Lemma 3.1. Then for any $m \geqq 0$ there exist a constant $C_{m, 1}$ and an integer $M(m)$ such that for any $\alpha_{0}, \alpha_{L}, \alpha_{j_{k}}$, $0 \leqq k \leqq s$, with $\left|\alpha_{j_{k}}\right| \leqq m,\left|\alpha_{L}\right| \leqq m,\left|\alpha_{0}\right| \leqq m$, 


$$
\begin{aligned}
& \left|\partial_{x_{L}}^{\alpha_{L}} \partial_{x_{0}}^{\alpha_{0}} \prod_{k=1}^{s} \partial_{x_{k}}^{\alpha_{j k}} b_{j_{s} j_{s-1} \cdots j_{1}}\left(x_{L}, x_{j_{s}}, x_{j_{s-1}}, \cdots, x_{j_{1}}, x_{0}\right)\right| \\
& \quad \leqq C_{m, 1}^{s}\left(\prod_{k=1}^{s} \nu^{-1} t_{j_{k}}\right)\|a\|_{M(m),\left\{j_{u}\right\}},
\end{aligned}
$$

where

$$
\left.\|a\|_{M(m),\left\{j_{u}\right\}}=\operatorname{Max} \sup \mid \partial_{x_{L}}^{\alpha_{L}} \partial_{x_{0}}^{\alpha_{0}} \prod_{k=1}^{s} \partial_{x_{j_{k}}}^{\beta_{j k}} \partial_{x_{j_{k}-1}}^{\beta_{j k}-1} a\left({\overline{x_{L}, x_{j_{s}}}}\right), \widetilde{x_{j_{s-1}}, x_{j s-1}}, \cdots, \widetilde{x_{j_{1}-1}, x_{0}}\right) \mid,
$$

where Max is taken over indices satisfying $\left|\beta_{j_{k}}\right|,\left|\beta_{j_{k}-1}\right| \leqq M(m)$ and sup is taken for $x_{j_{u}-1} \in \mathbb{R}, u=1, \cdots, s$. We can choose $M(m)=2 m+4+2$.

We assume Lemma 4.1 for the time being. Then we can prove Theorem 1. In fact, combining (4.7) and Lemma 4.1, we have

$$
\left|\partial_{x_{L}}^{\alpha_{L}} \partial_{x_{0}}^{\alpha_{0}} a_{j_{s} j_{s-1} \cdots j_{1}}\left(x_{L}, x_{0}\right)\right| \leqq C_{m}^{s} C_{m^{\prime}, 1}^{s}\left(\prod_{r=1}^{s} \nu^{-1} t_{j_{r}}\right)\|a\|_{M\left(m^{\prime}\right),\left\{j_{u}\right\}},
$$

where $m^{\prime}=K(m)$. On the other hand (H.2) implies that

$$
\|a\|_{M\left(m^{\prime}\right),\left\{j_{u}\right\}} \leqq A_{M\left(m^{\prime}\right)} X_{M\left(m^{\prime}\right)}^{s} .
$$

Therefore, we obtain from (4.14) that

$$
\begin{aligned}
\left|\partial_{x_{L}}^{\alpha_{L}} \partial_{x_{0}}^{\alpha_{0}} r\left(x_{L}, x_{0}\right)\right| & \leqq\left|\left(\sum^{\prime} C_{m}^{s} C_{m^{\prime}, 1}^{s} X_{M\left(m^{\prime}\right)}^{s} \prod_{r=1}^{s}\left(\mathcal{\nu}^{-1} t_{j_{r}}\right)\right)\right| A_{M\left(m^{\prime}\right)} \\
& \leqq\left[\prod_{j=1}^{L}\left(1+C_{m} C_{m^{\prime}, 1} X_{M\left(m^{\prime}\right)} \mathcal{\nu}^{-1} t_{j}\right)-1\right] A_{M\left(m^{\prime}\right)} .
\end{aligned}
$$

We have proved our Theorem 1 up to the proof of Lemma 4.1. (Since we can choose $m^{\prime}=K(m)=10 m+10+20$, we choose $M\left(m^{\prime}\right)=50(m+1+1)$ ).

Lemma 4.1 follows immediately from the next

Lemma 4.2. We assume (H.1) for the phase function. Let $a\left(x_{L}, x_{L-1}\right.$, $\left.\cdots, x_{1}, x_{0}\right)$ be a function of $L+1$ variables satisfying assumption (H.2). Then for any sequence of integers $0=k_{0}<k_{1}-1<k_{1}<k_{2}-1<\cdots$ $<k_{r}-1<k_{r}<k_{r+1}=L$ we introduce the function

$$
\begin{aligned}
& p_{k_{r} k_{r-1} \cdots k_{1}}\left(x_{L}, x_{L-1}, \cdots, x_{k_{r+1}}, x_{k_{r}}, x_{k_{r-1}}, \cdots, x_{k_{1}}, x_{0}\right) \\
& \quad=\left(Q_{k_{r}} Q_{k_{r}-1} \cdots Q_{1} a\right)\left(x_{L}, \cdots, x_{k_{r}+1}, x_{k_{r}}, x_{k_{r-1}}, \cdots, x_{k_{1}}, x_{0}\right),
\end{aligned}
$$

where $\quad Q_{j}=\mathrm{Id} \quad$ for $j=k_{r}, k_{r-1}, \cdots, k_{1}$,

$$
=R_{j} \quad \text { for } j=k_{r}-1, k_{r-1}-1, \cdots, k_{1}-1 \text {, }
$$

$=S_{j} \quad$ otherwise. 
This function enjoys the following estimate: For any $m \geqq 0$, there exist constants $C_{m, 2}$ and $M(m)$ such that if $\left|\alpha_{L}\right|,\left|\alpha_{0}\right|$ and $\left|\alpha_{k_{j}}\right| \leqq m,(j=1,2, \cdots, r)$,

$$
\begin{aligned}
& \left|\left(\prod_{j=0}^{r+1} \partial_{x_{k j}}^{\alpha_{k i}}\right) p_{k_{r} k_{r-1} \cdots k_{1}}\left(\widetilde{x_{L}, x_{k_{r}}}, x_{k_{r-1} \ldots}, x_{k_{1}}, x_{0}\right)\right| \\
& \leqq C_{m, 2}^{r} \prod_{j=1}^{r}\left(\frac{t_{k_{j}} T\left(k_{j}-1, k_{j-1}+1\right)}{\nu T\left(k_{j}, k_{j-1}+1\right)}\right) \\
& \times \max \sup \left|\partial_{x_{L}}^{\alpha_{L}} \partial_{x_{0}}^{\beta_{0}}\left(\prod_{j=1}^{r} \partial_{x_{k_{j}}}^{\beta_{k_{j}}} \partial_{x_{k_{j}-1}}^{\beta_{k_{j}-1}}\right) a\left(\widetilde{x_{L}, x_{k_{r}}}, \widetilde{x_{k_{r}-1}, x_{k_{r-1}}}, \cdots, \overline{x_{k_{1}-1}, x_{0}}\right)\right| \text {, }
\end{aligned}
$$

where max is taken over those indices $\beta_{k_{j}} \leqq \alpha_{k_{j}},\left|\beta_{k_{j}-1}\right| \leqq M(m), j=0,1, \cdots, r$ and sup is taken with respect to $x_{k_{j-1}} \in \mathbb{R}, j=1, \cdots, r$. Moreover, for any integers $l_{1}, \cdots, l_{q}$ with $k_{r}<l_{1}-1<l_{1}<l_{2}-1<\cdots<l_{q} \leqq L-1$, for arbitrary multi-indices $\alpha_{l_{u}}, \alpha_{l_{u}-1}(1 \leqq u \leqq q)$ and for multi-indices $\alpha_{k_{j}}$ with $\left|\alpha_{k_{j}}\right| \leqq m(0 \leqq j \leqq r+1)$, we have

$$
\begin{aligned}
& \mid \partial_{x_{L}}^{\alpha_{L}} \partial_{x_{0}}^{\alpha_{0}} \prod_{u=1}^{q}\left(\partial_{x_{l}}^{\alpha} l_{u} \partial_{x_{u}-1}^{\alpha_{l} l_{u-1}}\right) \prod_{j=1}^{r}\left(\partial_{x_{k j}}^{\alpha_{k_{j}}}\right) \\
& \times p_{k_{r} k_{r-1} \cdots k_{1}}\left({\widetilde{x_{L}, x_{l_{q}}}}_{x_{l_{q}-1}}, \cdots,{\widetilde{x_{l_{1}-1}, x_{k_{r}}}}_{x_{k_{r-1}}}, \cdots, x_{0}\right) \\
& \leqq C_{m, 2}^{r} \prod_{u=1}^{r}\left(\frac{t_{k_{u}} T\left(k_{u}-1, k_{u-1}+1\right)}{\nu T\left(k_{u}, k_{u-1}+1\right)}\right) \\
& \times \max \sup \mid \partial_{x_{L}}^{\alpha_{L}} \partial_{x_{0}}^{\beta_{0}} \prod_{u=1}^{q}\left(\partial_{x_{l_{u}}}^{\alpha_{l}} \partial_{x_{l_{u}-1}}^{\alpha_{l_{u}-1}}\right) \prod_{u=1}^{r}\left(\partial_{x_{k_{u}}}^{\beta_{k_{u}}} \partial_{x_{k_{u}-1}}^{\beta_{k_{u}-1}}\right)
\end{aligned}
$$

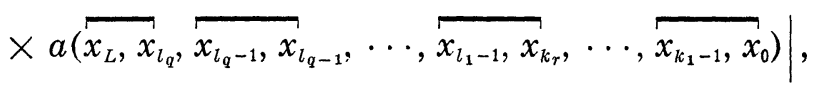

where max is taken over those indices which satisfy, $\beta_{k_{u}} \leqq \alpha_{k_{u}},\left|\beta_{k_{u}-1}\right| \leqq M(m)$, $(u=1,2, \cdots, r)$ and $\beta_{0} \leqq \alpha_{0}$; sup is taken with respect to $x_{k_{u}-1} \in \mathbb{R}, u=1$, $\cdots, r$. Constants $C_{m, 2}$ and $M(m)$ depend only on $m$. We can choose $M(m)=2 m+4+2$.

Proof. We prove by induction on $r$. The case of $r=1$. We abbreviate $k_{1}$ as $k$.

If $k \geqq 3$, then $p_{k}\left(x_{L}, \cdots, x_{k+1}, x_{k}, x_{0}\right)=R_{k-1} S_{k-2}, \cdots, S_{1} a\left(x_{L}, \cdots, x_{k}, x_{0}\right)$.

If $k=2$, then $p_{k}\left(x_{L}, \cdots, x_{k+1}, x_{k}, x_{0}\right)=R_{1} a\left(x_{L}, \cdots, x_{2}, x_{0}\right)$.

We set

$$
\begin{aligned}
q\left(x_{L}, \cdots, x_{k}, x_{k-1}, x_{0}\right) & =S_{k-2}, \cdots, S_{1} a\left(x_{L}, \cdots, x_{k}, x_{k-1}, x_{0}\right), \text { if } k \geqq 3, \\
& =a\left(x_{L}, \cdots, x_{2}, x_{1}, x_{0}\right), \quad \text { if } k=2 .
\end{aligned}
$$

Let $S_{1,1}^{\sharp}\left(x_{1}, x_{0}\right)=S_{1}\left(t_{1}, x_{1}, x_{0}\right)$. Then $p_{k}$ is defined by the equality: 


$$
\begin{aligned}
\left(\frac{E}{t_{k}}\right)^{1 / 2}\left(\frac{E}{T(k-1,1)}\right)^{1 / 2} \int_{\mathrm{R}} e^{-i_{\nu}\left(S_{k}\left(t_{k}, x_{k}, x_{k-1}\right)+S_{k-1,1}^{\#}\left(x_{k-1}, x_{0}\right)\right)} & \times q\left(x_{L}, \cdots, x_{k}, x_{k-1}, x_{0}\right) d x_{k-1} \\
= & \left(\frac{E}{T(k, 1)}\right)^{1 / 2} e^{-i \nu S_{k, 1}^{\#}\left(x_{k}, x_{0}\right)} \\
& \times\left[D\left(x_{k}, x_{0}\right)^{-1 / 2} q\left(x_{L}, \cdots, \widehat{x_{k}, x_{0}}\right)+p_{k}\left(x_{L}, \cdots, x_{k+1}, x_{k}, x_{0}\right)\right] .
\end{aligned}
$$

Therefore,

$$
\begin{aligned}
& \left(\frac{E}{t_{k}}\right)^{1 / 2}\left(\frac{E}{T(k-1,1)}\right)^{1 / 2} \int_{\mathrm{R}} e^{-i_{\nu}\left(S_{k}\left(t_{k}, x_{k}, x_{k-1}\right)+S_{k-1,1}^{\#}\left(x_{k-1}, x_{0}\right)\right)} \\
& \quad \times q\left(\widetilde{x_{L}, x_{k}}, x_{k-1}, x_{0}\right) d x_{k-1} \\
& =\left(\frac{E}{T(k, 1)}\right)^{1 / 2} e^{-i_{\nu} S_{k, 1}^{\sharp}\left(x_{k}, x_{0}\right)}\left(D\left(x_{k}, x_{0}\right)^{-1 / 2} q\left(\bar{x}_{L}, x_{k}, x_{0}\right)+p_{k}\left(x_{L}, x_{k}, x_{0}\right)\right) .
\end{aligned}
$$

Similarly, if $k<l_{1}-1<l_{1}<l_{2}-1<\cdots<l_{q} \leqq L-1$, then

$$
\begin{aligned}
& \left(\frac{E}{t_{k}}\right)^{1 / 2}\left(\frac{E}{T(k-1,1)}\right)^{1 / 2} \int_{\mathrm{R}} e^{-i_{\nu}\left(S_{k}\left(t_{k}, x_{k}, x_{k-1}\right)+S_{k-1,1}^{\#}\left(x_{k-1}, x_{0}\right)\right)} \\
& \times q\left(\widehat{x}_{L}, x_{l_{q}},{\overparen{x_{l_{q}-1},}}_{l_{q-1}}, \cdots,{\overparen{x_{l_{1}-1},}}_{x_{k}}, x_{k-1}, x_{0}\right) d x_{k-1} \\
& =\left(\frac{E}{T(k, 1)}\right)^{1 / 2} e^{-i \nu S_{k, 1}^{\#}\left(x_{k}, x_{0}\right)} \\
& \times\left(D\left(x_{k}, x_{0}\right)^{-1 / 2} q\left({\overrightarrow{x_{L}, x_{l_{q}}}}_{x_{l_{q}-1}, x_{l_{q-1}}}, \cdots, \widetilde{x_{l_{1-1}}, x_{k}, x_{0}}\right)\right.
\end{aligned}
$$

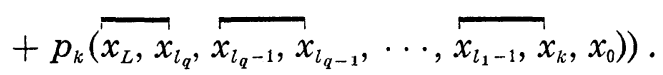

We prove (4.18) for $r=1$. Differentiating both sides of (4.21) with respect to $x_{L}$ and applying the stationary phase method Lemma 3.5 to (4.21), we obtain the estimate for $p_{k}$ : For any $m$ there exists $C_{m, 0}$ such that if $\left|\alpha_{k}\right|,\left|\alpha_{0}\right| \leqq m$ and $\alpha_{L}$ is arbitrary,

$$
\begin{aligned}
\left|\partial_{x_{L}}^{\alpha_{L}} \partial_{x_{k}}^{\alpha_{k}} \partial_{x_{0}}^{\alpha_{0}} p_{k}\left(x_{L}, x_{k}, x_{0}\right)\right| \leqq C_{m, 0} \frac{t_{k} T(k-1,1)}{T(k, 1) \nu} \\
\times \max \sup _{x_{k-1}}\left|\partial_{x_{L}}^{\alpha_{L}} \partial_{x_{k}}^{\beta_{k}} \partial_{x_{k-1}}^{\beta_{k-1}} \partial_{x_{0}}^{\beta_{0}} q\left(\overline{x_{L}, x_{k}}, x_{k-1}, x_{0}\right)\right|,
\end{aligned}
$$

here max is taken over those indices for which $\beta_{k} \leqq \alpha_{k},\left|\beta_{k-1}\right| \leqq 2 m+4+2$ and $\beta_{0} \leqq \alpha_{0}$. Since (4.20) implies

$$
\text { (4.24) } \begin{aligned}
q\left(x_{L}, \cdots, x_{k}, x_{k-1}, x_{0}\right) & =D\left(x_{k-1}, x_{0}\right)^{-1 / 2} a\left(x_{L}, \cdots, x_{k}, \widetilde{x_{k-1}, x_{0}}\right) & & \text { if } k \geqq 3 \\
& =a\left(x_{L}, \cdots, x_{2}, x_{1}, x_{0}\right) & & \text { if } k=2 .
\end{aligned}
$$


Leibnitz' rule gives

$$
\begin{aligned}
& \left|\partial_{x_{L}}^{\alpha_{L}} \partial_{x_{k}}^{\alpha_{k}} \partial_{x_{0}}^{\alpha_{0}} p_{k}\left(\widetilde{x_{L}, x_{k}}, x_{0}\right)\right| \\
& \quad<C_{m, 0} C_{m, 3}\left(\frac{t_{k} T(k-1,1)}{\nu T(k, 1)}\right) \max \sup _{x_{k-1}}\left|\partial_{x_{L}}^{\alpha_{L}} \partial_{x_{k}}^{\beta_{k}} \partial_{x_{k-1}}^{\beta_{k}-1} \partial_{k_{0}}^{\beta_{0}} a\left(\widetilde{x_{L}, x_{k}}, \widetilde{x_{k-1}, x_{0}}\right)\right|
\end{aligned}
$$

Here $C_{m, 3}=2^{(2 m+4+2)}$, max is taken for $\beta_{k} \leqq \alpha_{k},\left|\beta_{k-1}\right| \leqq 2 m+4+2$ and $\beta_{0} \leqq \alpha_{0}$. Choose $M(m)$ and $C_{m, 2}$ so that

$$
M(m)=2 m+4+2 \text { and } C_{m, 2} \geqq C_{m, 0} C_{m, 3} .
$$

Then this proves estimate (4.18) for $r=1$.

We prove (4.19) for $r=1$. Using the stationary phase method to (4.22) and using (4.24) again, we obtain the following estimate: For any $m \geqq 0$ if $\left|\alpha_{k}\right|,\left|\alpha_{0}\right| \leqq m$ and $\alpha_{l_{u}}, \alpha_{l_{u}-1},(u=1, \cdots, q), \alpha_{L}$ are arbitrary multiindices,

$$
\begin{aligned}
& \left|\partial_{x_{L}}^{\alpha_{L}} \partial_{x_{k}}^{\alpha_{k}} \partial_{x_{0}}^{\alpha_{0}} \prod_{u=1}^{q}\left(\partial_{x_{l_{u}}}^{\alpha_{l} l_{u}} \partial_{x_{l_{u}-1}}^{\alpha_{l_{u}-1}}\right) p_{k}\left(\widetilde{x_{L}, x_{l_{q}}}, \widetilde{x_{l_{q}-1}, x_{l_{q-1}}}, \cdots, \widetilde{x_{l_{1}-1}, x_{k}}, x_{0}\right)\right| \\
& \leqq C_{m, 0}\left(\frac{t_{k} T(k-1,1)}{\nu T(k, 1)}\right) \max \sup _{x_{k-1}} \mid \partial_{x_{L}}^{\alpha_{L}} \partial_{x_{k}}^{\beta_{k}} \partial_{x_{k-1}}^{\beta_{k-1}} \partial_{x_{0}}^{\beta_{0}} \prod_{u=1}^{q} \partial_{x_{l_{u}}}^{\alpha_{l} l_{u}} \partial_{x_{l_{u}-1}^{\alpha} l_{u-1}}^{\alpha_{u}}
\end{aligned}
$$

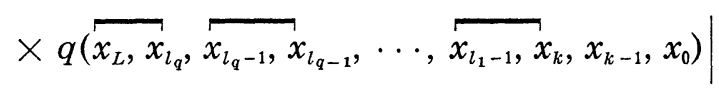

$$
\begin{aligned}
& \leqq C_{m, 0} C_{m, 3}\left(\frac{t_{k} T(k-1,1)}{\nu T(k, 1)}\right) \max \sup _{x_{k-1}} \mid \partial_{x_{L}}^{\alpha_{L}} \partial_{x_{k}}^{\beta_{k}} \partial_{x_{k-1}}^{\beta_{k-1}^{\prime}} \partial_{x_{0}}^{\beta_{0}^{\prime}}\left(\prod_{u=1}^{q} \partial_{x_{l_{u}}}^{\alpha_{l} \partial_{x_{u}}} \partial_{x_{u-1}}^{\alpha_{l_{u}-1}}\right) \\
& \times a\left({\widetilde{x_{L}, x_{l_{q}}}}_{x_{l_{q}-1}, x_{l_{q-1}}}, \cdots,{\widetilde{x_{l_{1}-1}, x_{k}}}_{x_{k-1}, x_{0}}\right) \mid .
\end{aligned}
$$

Max is taken for $\beta_{k} \leqq \alpha_{k}, \beta_{0} \leqq \alpha_{0}$ and $\left|\beta_{k-1}\right| \leqq 2 m+4+2$ in the middle term. In the last term max is taken for $\beta_{k} \leqq \alpha_{k}, \beta_{0}^{\prime} \leqq \alpha_{0}$ and $\left|\beta_{k-1}^{\prime}\right| \leqq$ $2 m+4+2$. We choose $M(m)$ and $C_{m, 2}$ as in (4.25). Then (4.19) of the case $r=1$ is proved.

Now assuming Lemma 4.2 for $r$, we prove (4.18) for $r+1$.

Let $k_{r+1}$ be any integer such that $L>k_{r+1}-1>k_{r}$ and we let

$$
\begin{aligned}
& p_{k_{r+1} k_{r} \cdots k_{1}}\left(x_{L}, \cdots, x_{k_{r+1}+1}, x_{k_{r+1}}, x_{k_{r}}, \cdots, x_{k_{1}}, x_{0}\right) \\
& \quad=R_{k_{r+1}-1} \cdots R_{k_{r-1}} \cdots R_{k_{1-1}} \cdots a\left(x_{L}, \cdots, x_{k_{r+1}+1}, x_{k_{r+1}}, x_{k_{r}}, \cdots, x_{k_{1}}, x_{0}\right) .
\end{aligned}
$$

Set

(4.27) $q\left(x_{L}, \cdots, x_{k_{r+1}}, x_{k_{r+1}-1}, x_{k_{r}}, \cdots, x_{k_{1}}, x_{0}\right)$

$$
=S_{k_{r+1}-2} \cdots S_{k_{r+1}} p_{k_{r} \cdots k_{1}}\left(x_{L}, \cdots, x_{k_{r+1}}, x_{k_{r+1}-1}, x_{k_{r}}, \cdots, x_{k_{1}}, x_{0}\right) \text {. }
$$


Then $p_{k_{r+1} k_{r} \cdots k_{1}}$ is defined by the equality

$$
\begin{aligned}
& \left(\frac{E}{t_{k_{r+1}}}\right)^{1 / 2}\left(\frac{E}{T\left(k_{r+1}-1, k_{r}+1\right)}\right)^{1 / 2}
\end{aligned}
$$

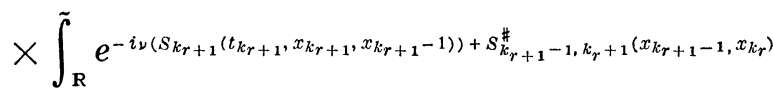

$$
\begin{aligned}
& \times q\left(x_{L}, \cdots, x_{k_{r+1}}, x_{k_{r+1}-1}, x_{k_{r}}, x_{k_{r-1}}, \cdots, x_{k_{1}}, x_{0}\right) d x_{k_{r+1}-1}
\end{aligned}
$$

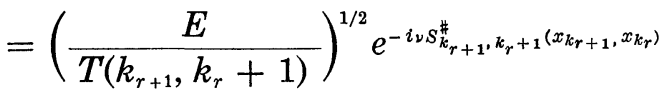

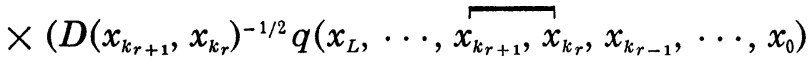

$$
\begin{aligned}
& \left.+p_{k_{r+1} \cdots k_{1}}\left(x_{L}, \cdots, x_{k_{r+1}+1}, x_{k_{r+1}}, x_{k_{r}}, x_{k_{r-1}}, \cdots, x_{k_{1}}, x_{0}\right)\right) \text {. }
\end{aligned}
$$

Therefore,

$$
\begin{aligned}
& \left(\frac{E}{t_{k_{r+1}}}\right)^{1 / 2}\left(\frac{E}{T\left(k_{r+1}-1, k_{r}+1\right)}\right)^{1 / 2} \\
& \times \int_{\mathbf{R}} e^{-i_{\nu}\left(S_{k_{r+1}}\left(t_{k_{r+1}}, x_{k_{r+1}}, x_{k_{r+1}-1}\right)+S_{k_{r+1}-1, k_{r}+1}^{\#}\left(x_{k_{r+1}}-1, x_{k_{r}}\right)\right)} \\
& \times q\left({\overline{x_{L},}}_{k_{r+1}}, x_{k_{r+1}-1}, x_{k_{r}}, \cdots, x_{k_{1}}, x_{0}\right) d x_{k_{r+1}-1} \\
& =\left(\frac{E}{T\left(k_{r+1}, k_{r}+1\right)}\right)^{1 / 2} e^{-i \nu S_{k_{r+1}}^{\#}, k_{r}+1}\left(x_{k_{r+1}}, x_{k_{r}}\right.
\end{aligned}
$$

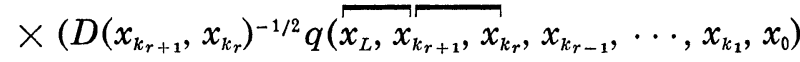

$$
\begin{aligned}
& \left.+p_{k_{r+1} k_{r} \cdots k_{1}}\left(\vec{x}_{L}, x_{k_{r+1}}, x_{k_{r}}, x_{k_{r-1}}, \cdots, x_{k_{1}}, x_{0}\right)\right) \text {. }
\end{aligned}
$$

Apply the stationary phase method Lemma 3.5 to (4.28). Then for any $m \geqq 0$ if $\left|\alpha_{k_{r}}\right|$ and $\left|\alpha_{k_{r+1}}\right| \leqq m$, we have with the same $C_{m, 0}$ as in (4.23)

$$
\begin{aligned}
& \left|\partial_{x_{L}}^{\alpha_{L}} \partial_{x_{k_{r+1}}}^{\alpha_{k_{r+1}}} \partial_{x_{k_{r}}}^{\alpha_{k_{r}}} \partial_{x_{0}}^{\alpha_{0}}\left(\prod_{u=1}^{r-1} \partial_{x_{k_{u}}}^{\alpha_{k_{u}}}\right) p_{k_{r+1} \cdots k_{1}}\left(x_{L}, x_{k_{r+1}}, x_{k_{r}}, \cdots, x_{k_{1}}, x_{0}\right)\right| \\
& <C_{m, 0}\left(\frac{t_{k_{r+1}} T\left(k_{r+1}-1, k_{r}+1\right)}{\nu T\left(k_{r+1}, k_{r}+1\right)}\right) \\
& \times \max \sup _{x_{k_{r+1}-1}} \mid \partial_{x_{L}}^{\alpha_{L}} \partial_{x_{k_{r}+1}}^{\beta_{k_{r+1}}} \partial_{x_{k_{r}+1}-1}^{\beta_{k_{r+1}+1}} \partial_{x_{k_{r}}}^{\beta_{k_{r}}} \partial_{x_{0}}^{\alpha_{0}} \prod_{u=1}^{r-1} \partial_{x_{k_{u}}}^{\alpha_{k_{u}}} \\
& \times q\left(\bar{x}_{L}, x_{k_{r+1}}, x_{k_{r+1}-1}, x_{k_{r}}, \cdots, x_{k_{1}}, x_{0}\right) \mid,
\end{aligned}
$$

$\max$ is taken for $\beta_{k_{r+1}} \leqq \alpha_{k_{r+1}}, \beta_{k_{r}} \leqq \alpha_{k_{r}}$ and $\left|\beta_{k_{r+1}-1}\right| \leqq 2 m+4+2$. Here $\alpha_{0}, \alpha_{k_{u}}(u=1,2, \cdots, r-1)$ and $\alpha_{L}$ are arbitrary multi-indices.

On the other hand, by definition (4.27) we have 


$$
\begin{aligned}
& q\left(x_{L}, \cdots, x_{k_{r+1}}, x_{k_{r+1}-1}, x_{k_{r}}, \cdots, x_{k_{1}}, x_{0}\right) \\
& \quad=D\left(x_{k_{r+1}-1}, x_{k_{r}}\right)^{-1 / 2} p_{k_{r} \cdots k_{1}}\left(x_{L}, \cdots, x_{k_{r+1}}, \bar{x}_{k_{r+1}-1}, x_{k_{r}}, \cdots, x_{k_{1}}, x_{0}\right) .
\end{aligned}
$$

Therefore,

$$
\begin{aligned}
& q\left(\bar{x}_{L}, x_{k_{r+1}}, x_{k_{r+1}-1}, x_{k_{r}}, \cdots, x_{k_{1}}, x_{0}\right) \\
& \quad=D\left(x_{k_{r+1}-1}, x_{k_{r}}\right)^{-1 / 2} p_{k_{r} \cdots k_{1}}\left(x_{L}, x_{k_{r+1}},{\left.\overparen{x_{k_{r+1}-1}, x_{k_{r}}}, \cdots, x_{k_{1}}, x_{0}\right) .}^{\quad}\right.
\end{aligned}
$$

And we have

$$
\begin{aligned}
& \left|\partial_{x_{L}}^{\alpha_{L}} \partial_{x_{k_{r+1}}}^{\alpha_{k_{r}+1}} \partial_{x_{k_{r}}}^{\alpha_{k_{r}}} \partial_{x_{0}}^{\alpha_{0}}\left(\prod_{u=1}^{r-1} \partial_{x_{k_{u}}}^{\alpha_{k_{u}}}\right) p_{k_{r+1} \ldots k_{1}}\left(x_{L}, x_{k_{r+1}}, x_{k_{r}}, \cdots, x_{k_{1}}, x_{0}\right)\right| \\
& \leqq C_{m, 0} C_{m, 3}\left(\frac{t_{k_{r+1}} T\left(k_{r+1}-1, k_{r}+1\right)}{\nu T\left(k_{r+1}, k_{r}+1\right)}\right)
\end{aligned}
$$

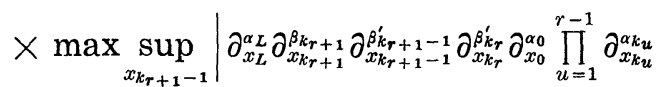

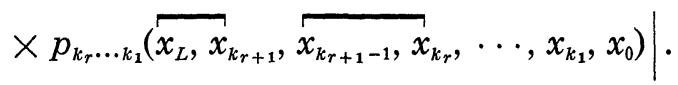

Here max is taken for multi-indices $\left|\beta_{k_{r+1}-1}^{\prime}\right| \leqq 2 m+4+2$, and $\beta_{k_{r}}^{\prime} \leqq \beta_{k_{r}}$. Now we restrict to the case $\left|\alpha_{k_{j}}\right| \leqq m, j=1, \cdots, r$. We can apply induction hypothesis (4.19) for $r$ with $q=1, l_{1}=k_{r+1}, \alpha_{l_{1}}=\beta_{k_{r+1}}$ and $\alpha_{l_{1}-1}=\beta_{k_{r+1}-1}$ to (4.33) and we get a majorization for the right hand side of (4.33). Consequently, if $\left|\alpha_{k}\right|,\left|\alpha_{k_{j}}\right|,\left|\alpha_{0}\right| \leqq m$, we have

$$
\begin{aligned}
& \left|\partial_{x_{L}}^{\alpha_{L}} \partial_{x_{r+1}}^{\alpha_{k_{r+1}}} \partial_{x_{k_{r}}}^{\alpha_{k_{r}}} \partial_{x_{0}}^{\alpha_{0}}\left(\prod_{u=1}^{r-1} \partial_{x_{k_{u}}}^{\alpha_{k_{u}}}\right) p_{k_{r+1} \cdots k_{1}}\left(\widehat{x}_{L}, x_{k_{r+1}}, \widetilde{x_{k_{r+1}-1}, x_{k_{r}}}, \cdots, x_{k_{1}}, x_{0}\right)\right| \\
& \leqq C_{m, 0} C_{m, 3} C_{m, 2}^{r}\left(\frac{t_{k_{r+1}} T\left(k_{r+1}-1, k_{r}+1\right)}{\nu T\left(k_{r+1}, k_{r-1}+1\right)}\right) \prod_{u=1}^{r}\left(\frac{t_{k_{u}} T\left(k_{u}-1, k_{u-1}+1\right)}{\nu T\left(k_{u}, k_{u-1}+1\right)}\right)
\end{aligned}
$$

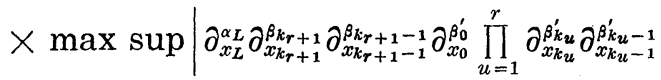

$$
\begin{aligned}
& \times a\left({\widehat{x_{L}, x_{k_{r+1}}}}_{x_{k_{r+1}-1}, x_{k_{r}}}, \cdots,{\widetilde{x_{k_{1}-1}, x_{0}}}\right) \text {. }
\end{aligned}
$$

Here max is taken for $\beta_{0}^{\prime} \leqq \alpha_{0}, \beta_{k_{r+1}} \leqq \alpha_{k_{r+1}},\left|\beta_{k_{r+1}-1}\right| \leqq 2 m+4 d+2, \beta_{k_{u}}^{\prime} \leqq \alpha_{k_{u}}$, $\left|\beta_{k_{u}-1}^{\prime}\right| \leqq 2 m+4+2,(u=1, \cdots, r)$ and sup is taken with respect to $x_{k_{u}-1} \in \mathbb{R}, u=1, \cdots, r+1$. We may choose $M(m)$ and $C_{m, 2}$ as in (4.25). We have proved (4.18) for $r+1$.

We next prove (4.19) for $r+1$. Let $l_{1}, l_{2}, \ldots, l_{q}$ be a sequence of integers with the property $k_{r}<k_{r+1}-1<k_{r+1}<l_{1}-1<l_{1}<\ldots<l_{q}-1$ $<l_{q} \leqq L-1$. Then we have, from (4.28), 


$$
\begin{aligned}
& \left(\frac{E}{t_{k_{r+1}}}\right)^{1 / 2}\left(\frac{E}{T\left(k_{r+1}-1, k_{r}+1\right)}\right)^{1 / 2} \\
& \times \int_{R} e^{-i \nu\left(S_{k_{r+1}}\left(t_{k_{r+1}}, x_{k_{r+1}}, x_{k_{r+1}-1}\right)+S_{k_{r+1}-1, k_{r+1}}^{\#}\left(x_{k_{r+1}-1}, x_{k_{r}}\right)\right)} \\
& \times q\left(\bar{x}_{L}, x_{l_{q}},{\widetilde{x_{l_{q}-1}}, x_{l_{q-1}}}, \cdots,{\widehat{x_{l_{1}-1},}}_{k_{k_{r+1}}}, x_{k_{r+1}-1}, \cdots, x_{k_{1}}, x_{0}\right) d x_{k_{r+1}-1} \\
& =\left(\frac{E}{T\left(k_{r+1}, k_{r}+1\right)}\right)^{1 / 2} e^{-i \nu S_{k_{r+1}}^{\#}, k_{r}+1}\left(x_{k_{r+}}, x_{k_{r}}\right) \\
& \times\left(D ( x _ { k _ { r + 1 } } , x _ { k _ { r } } ) ^ { - 1 / 2 } q \left({\widetilde{x_{L}, x_{l_{q}}}}_{x_{l_{q-1}}, x_{l_{q-1}}, \cdots},\right.\right.
\end{aligned}
$$

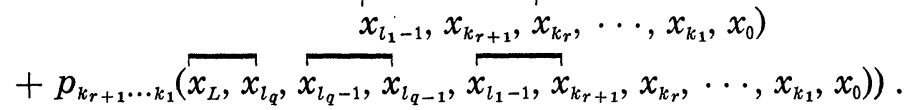

We apply the stationary phase method Lemma 3.5 to (4.34). For any $m \geqq 0$ let $\alpha_{k_{r}}$ and $\alpha_{k_{r+1}}$ be two multi-indices with $\left|\alpha_{k_{r}}\right|,\left|\alpha_{k_{r+1}}\right| \leqq m$; let $\alpha_{k_{u}}$ $(u=1,2, \cdots, r-1), \alpha_{L}, \alpha_{0}$, and $\alpha_{l_{u}}(u=1,2, \cdots, q)$ be arbitrary multiindices. Then with the same constant $C_{m, 0}$ as in (4.23) and (4.30), we have

$$
\begin{aligned}
& \mid \partial_{x_{L}}^{\alpha_{L}} \partial_{x_{0}}^{\alpha_{0}}\left(\prod_{u=1}^{q} \partial_{x_{u_{u}}}^{\alpha_{l_{u}}} \partial_{x_{l_{u}-1}}^{\alpha_{l_{u}-1}}\right) \prod_{u=1}^{r+1} \partial_{x_{k_{u}}}^{\alpha_{k_{u}}}
\end{aligned}
$$

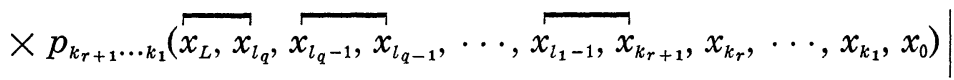

$$
\begin{aligned}
& \leqq C_{m, 0}\left(\frac{t_{k_{r+1}} T\left(k_{r+1}-1, k_{r}+1\right)}{\nu T\left(k_{r+1}, k_{r}+1\right)}\right)
\end{aligned}
$$

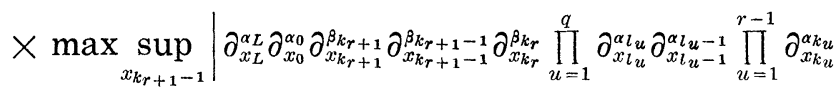

$$
\begin{aligned}
& \times q\left(x_{L}, x_{l_{q}},{ }_{l_{q-1}}, x_{l_{q-1}}, \cdots, x_{l_{1-1}}, x_{k_{r+1}}, x_{k_{r+1}-1}, x_{k_{r}}, \cdots, x_{k_{1}}, x_{0}\right) \mid \text {. }
\end{aligned}
$$

We use the relationship (4.31) between $q$ and $p_{k_{r} \ldots k_{1}}$. The right hand side of this inequality is majorized by

$$
\begin{aligned}
& C_{m, 0} C_{m, 3}\left(\frac{t_{k_{r+1}} T\left(k_{r+1}-1, k_{r}+1\right)}{\nu T\left(k_{r+1}, k_{r}+1\right)}\right)
\end{aligned}
$$

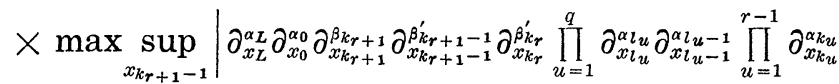

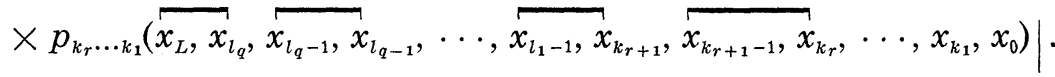

Here max is taken for $\beta_{k_{r+1}} \leqq \alpha_{k_{r+1}},\left|\beta_{k_{r+1}-1}^{\prime}\right| \leqq 2 m+4+2$ and $\beta_{k_{r}}^{\prime} \leqq \alpha_{k_{r}}$.

Now we assume that $\left|\alpha_{k_{j}}\right| \leqq m, j=1, \cdots, r+1$, and $\left|\alpha_{0}\right|,\left|\alpha_{L}\right| \leqq m$ as in Lemma 4.2 but that $\alpha_{l_{u}}, u=1, \cdots, q$, are arbitrary. Then we use 
the induction hypothesis (4.19) for $r$ where $q$ is replaced by $q+1$ and $\left(l_{1}, \cdots, l_{q}\right)$ is replaced by $\left(k_{r+1}, l_{1}, \cdots, l_{q}\right)$ to majorize (4.36). Then we have

$$
\begin{aligned}
& \mid \partial_{x_{L}}^{\alpha_{L}} \partial_{x_{0}}^{\alpha_{0}}\left(\prod_{u=1}^{q} \partial_{x_{u}}^{\alpha_{l_{u}}} \partial_{x_{l_{u}-1}}^{\alpha_{l_{u}-1}}\right) \prod_{u=1}^{r} \partial_{x_{k_{u}}}^{\alpha_{k_{u}}}
\end{aligned}
$$

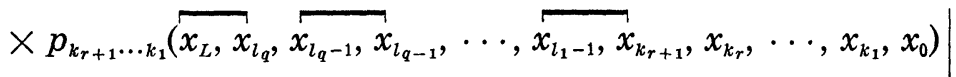

$$
\begin{aligned}
& \leqq C_{m, 0} C_{m, 3} C_{m, 2}^{r} \prod_{u=1}^{r+1}\left(\frac{t_{k_{u}} T\left(k_{u}-1, k_{u-1}+1\right)}{\nu T\left(k_{u}, k_{u-1}+1\right)}\right)
\end{aligned}
$$

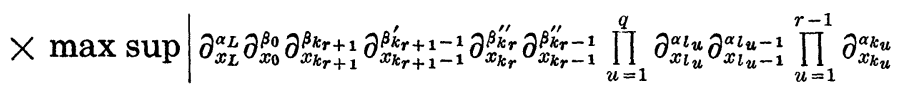

$$
\begin{aligned}
& \times a\left({\widetilde{x_{L}, x_{l_{q}}}}_{x_{l_{q}-1}, x_{l_{q-1}}}, \cdots,{\widetilde{x_{l_{1}-1}, x_{k_{r+1}}}}_{x_{k_{r+1}-1}, x_{k_{r}}}, \cdots,{\widetilde{x_{k_{1}}}, x_{0}}\right)
\end{aligned}
$$

$\max$ is taken for $\beta_{k_{r+1}} \leqq \alpha_{k_{r+1}},\left|\beta_{k_{r+1}-1}^{\prime}\right| \leqq 2 m+4 d+2, \beta_{k_{r}}^{\prime \prime} \leqq \alpha_{k_{r}},\left|\beta_{k_{r}-1}^{\prime \prime}\right|<$ $2 m+4+2, \beta_{0} \leqq \alpha_{0}$ and sup is taken with respect to $x_{k_{u}-1} \in \mathbb{R}, u=1,2$, $\cdots, r+1$. We can choose $M(m)$ and $C_{m, 2}$ as in (4.25). Then the above inequality proves (4.19) for $r+1$. We have completed proof of Lemma 4.2.

Proof of Theorem 1 has been completed.

\section{§5. Proof of Theorem 2}

We can proceed just as in the proof of Theorem 1. And we have

$$
I\left(\left\{t_{j}\right\}, S, 1, \nu\right)\left(x_{L}, x_{0}\right)=A_{0}\left(x_{L}, x_{0}\right)+\sum^{\prime} A_{j_{s} j_{s-1} \cdots j_{1}}\left(x_{L}, x_{0}\right) .
$$

Here

$$
A_{0}\left(x_{L}, x_{0}\right)=\left(\frac{E}{T_{L}}\right)^{1 / 2} e^{-i_{\nu} S_{L, 1}^{\#}\left(x_{L}, x_{0}\right)} D\left(x_{L}, x_{0}\right)^{-1 / 2}
$$

is the main term; $\Sigma^{\prime}$ is the same as in $\S 4$ and

$$
\begin{aligned}
& A_{j_{s} j_{s-1} \ldots j_{1}}\left(x_{L}, x_{0}\right)=\prod_{k=1}^{s+1}\left(\frac{E}{T\left(j_{k}, j_{k-1}+1\right)}\right)^{1 / 2} \\
& \quad \times \int_{\mathrm{R}^{s}} e^{-i \nu s_{j_{s}}^{\#} \ldots j_{1}\left(x_{L}, x_{j_{s}}, \cdots, x_{j_{1}}, x_{0}\right.} b_{j_{s} \ldots j_{1}}\left(x_{L}, x_{j_{s}}, \cdots, x_{j_{1}}, x_{0}\right) \prod_{k=1}^{s} d x_{j_{k}},
\end{aligned}
$$

where

$$
S_{j_{s} j_{s-1} \cdots j_{1}}^{\#}\left(x_{L}, x_{j_{s}}, \cdots, x_{j_{1}}, x_{0}\right)=\sum_{k=1}^{s+1} S_{j_{k}, j_{k-1}+1}^{\#}\left(x_{j_{k}}, x_{j_{k-1}}\right)
$$

and 


$$
\begin{aligned}
& b_{j_{s} j_{s-1} \cdots j_{1}}\left(x_{L}, x_{j_{s}}, \cdots, x_{j_{1}}, x_{0}\right) \\
& \quad=\left(Q_{L-1} Q_{L-2}, \cdots, Q_{1} 1\right)\left(x_{L}, x_{j_{s}}, x_{j_{s-1}}, \cdots, x_{j_{1}}, x_{0}\right),
\end{aligned}
$$

where $\quad Q_{j}=\mathrm{Id} \quad$ if $j=j_{s}, j_{s-1}, \cdots, j_{1}$,

$=R_{j}$ if $j=j_{s}-1, j_{s-1}-1, \cdots, j_{1}-1$,

$=S_{j}$ otherwise.

Applying Lemma 3.1 to $A_{j_{s} j_{s-1} \cdots j_{1}}\left(x_{L}, x_{0}\right)$, we have

$$
A_{j_{s} \cdots j_{1}}\left(x_{L}, x_{0}\right)=\left(\frac{E}{T_{L}}\right)^{1 / 2} e^{-i \nu S_{L, 1}^{\#}\left(x_{L}, x_{0}\right)} a_{j_{s} \ldots j_{1}}\left(x_{L}, x_{0}\right) .
$$

Thus setting, just as in (4.14),

$$
r\left(x_{L}, x_{0}\right)=D\left(x_{L}, x_{0}\right)^{1 / 2}\left(\sum^{\prime} a_{f_{s} \cdots j_{1}}\left(x_{L}, x_{0}\right)\right),
$$

we have

$$
I\left(\left\{t_{j}\right\}, S, 1, \nu\right)=\left(\frac{E}{T_{L}}\right)^{1 / 2} e^{-i \nu S_{L, 1}^{\#}\left(x_{L}, x_{0}\right)} D\left(x_{L}, x_{0}\right)^{-1 / 2}\left(1+r\left(x_{L}, x_{0}\right)\right) .
$$

We have only to obtain estimate of $r\left(x_{L}, x_{0}\right)$ to prove Theorem 2 . By virtue of Lemma 3.1, we get estimates of $a_{f_{s} \cdots j_{1}}\left(x_{L}, x_{0}\right)$ : For any $m \geqq 0$ there exists $C_{m}$ and $K(m)$ such that if $\left|\alpha_{L}\right|,\left|\alpha_{0}\right| \leqq m$

$$
\begin{aligned}
& \left|\partial_{x_{L}}^{\alpha_{L}} \partial_{x_{0}}^{\alpha_{0}} a_{j_{s} \cdots j_{1}}\left(x_{L}, x_{0}\right)\right| \\
& \quad \leqq C_{m}^{s} \max \sup \left|\partial_{x_{L}}^{\alpha_{L}} \partial_{x_{0}}^{\beta_{0}} \prod_{r=1}^{s} \partial_{x_{j_{r}}}^{\beta_{j_{r}}} b_{j_{s} \ldots j_{1}}\left(x_{L}, x_{j_{s}}, x_{j_{s-1}}, \cdots, x_{j_{1}}, x_{0}\right)\right|
\end{aligned}
$$

$\max$ is taken over $\beta_{0} \leqq \alpha_{0}$ and $\left|\beta_{j_{r}}\right| \leqq K(m)$.

We wish to obtain estimate of $b_{j_{s} \cdots j_{1}}$. Consider $x_{L}, \cdots, x_{j_{s+1}}$ as parameters and set

$$
\begin{aligned}
& \tilde{b}_{j_{s} j_{s-1} \cdots j_{1}}\left(x_{L}, \cdots, x_{j_{s+1}}, x_{j_{s}}, x_{j_{s-1}}, \cdots, x_{j_{1}}, x_{0}\right) \\
& \quad=\left(Q_{j_{s}} Q_{j_{s}-1}, \cdots, Q_{1} 1\right)\left(x_{L}, \cdots, x_{j_{s}+1}, x_{j_{s}}, x_{j_{s-1}}, \cdots, x_{j_{1}}, x_{0}\right),
\end{aligned}
$$

where $\quad Q_{j}=$ Id $\quad$ if $j=j_{s}, j_{s-1}, \cdots, j_{1}$,

$$
\begin{aligned}
& =R_{j} \quad \text { if } j=j_{s}-1, j_{s-1}-1, \cdots, j_{1}-1, \\
& =S_{j} \quad \text { otherwise. }
\end{aligned}
$$

The next Lemma gives estimate of $\tilde{b}_{j_{s} \cdots j_{1}}$.

Lemma 5.1. Let $\delta$ be as in Theorem 1 and $T_{L}<\delta$. Then $\tilde{b}_{s_{s} j_{s-1} \cdots j_{1}}$ is independent of $x_{L}, \cdots, x_{j_{s+1}}$. It is of the form 


$$
\begin{array}{r}
\tilde{b}_{j_{s} j_{s-1} \ldots j_{1}}\left(x_{L}, \cdots, x_{j_{s+1}}, x_{j_{s}}, x_{j_{s-1}}, \cdots, x_{j_{1}}, x_{0}\right) \\
=\prod_{r=1}^{s} \frac{t_{j_{r}}}{\nu} T\left(j_{r}-1, j_{r-1}+1\right)^{2} p_{j_{r}}\left(x_{j_{r}}, x_{j_{r-1}}\right)
\end{array}
$$

where $p_{j_{r}}\left(x_{j_{r}}, x_{j_{r-1}}\right)$ satisfies the estimates

$$
\left|\partial_{x_{j_{r}}}^{\alpha} \partial_{x_{j_{r-1}}}^{\beta} p_{j_{r}}\left(x_{j_{r}}, x_{j_{r-1}}\right)\right| \leqq C_{\alpha \beta} \text { for any } \alpha \text { and } \beta \text {. }
$$

Here constant $C_{\alpha \beta}$ depends only on $\alpha$ and $\beta$.

We assume Lemma 5.1 for the moment and continue proof of Theorem 2. Since $b_{j_{s} j_{s-1} \cdots j_{1}}=S_{L-1} S_{L-2} \cdots S_{j_{s+1}} \tilde{b}_{j_{s} j_{s-1} \cdots j_{1}}$, we can apply Lemma 5.1 to (5.5) and obtain

$$
\begin{aligned}
& b_{j_{s} j_{s-1} \cdots j_{1}}\left(x_{L}, x_{j_{s}}, \cdots, x_{j_{1}}, x_{0}\right) \\
& \quad=D\left(x_{L}, x_{j_{s}}\right)^{-1 / 2} \prod_{r=1}^{s}\left(\frac{t_{j_{r}}}{\nu}\right) T\left(j_{r}-1, j_{r-1}+1\right)^{2} p_{j_{r}}\left(x_{j_{r}}, x_{j_{r-1}}\right) .
\end{aligned}
$$

Combining this with (5.8), we have

$$
\begin{aligned}
& \left|\partial_{x_{L}}^{\alpha_{L}} \partial_{x_{0}}^{\alpha_{0}} a_{j_{s} \ldots j_{1}}\left(x_{L}, x_{0}\right)\right|<C_{m}^{s} \max \sup \mid \partial_{x_{L}}^{\alpha_{L}} \partial_{x_{0}}^{\beta_{0}} \prod_{r=1}^{s} \partial_{x_{j_{r}}}^{\beta_{j_{r}}} D\left(x_{L}, x_{j_{s}}\right)^{-1 / 2} \\
& \quad \times \prod_{r=1}^{s}\left(\frac{t_{j_{r}}}{\nu} T\left(j_{r}-1, j_{r-1}+1\right)^{2} p_{j_{r}}\left(x_{j_{r}}, x_{j_{r-1}}\right)\right) \mid
\end{aligned}
$$

Therefore, for any $m \geqq 0$ we can find a constant $C_{m, 1}$ such that

$$
\left|\partial_{x_{L}}^{\alpha_{L}} \partial_{x_{0}}^{\alpha_{0}} a_{\jmath_{s} \ldots j_{1}}\left(x_{L}, x_{0}\right)\right| \leqq C_{m, 1}^{s} \prod_{r=1}^{s}\left(\nu^{-1} t_{j_{r}} T\left(j_{r}-1, j_{r-1}+1\right)^{2}\right)
$$

as far as $\left|\alpha_{L}\right|$ and $\left|\alpha_{0}\right| \leqq m$. This and (5.6) imply

$$
\begin{aligned}
\left|\partial_{x_{L}}^{\alpha_{L}} \partial_{x_{0}}^{\alpha_{0}} r\left(x_{L}, x_{0}\right)\right| & <\sum_{\left(j_{s} \cdots j_{1}\right)}^{\prime} C_{m, 1}^{s} \prod_{r=1}^{s} \nu^{-1} t_{j_{r}} T\left(j_{r}-1, j_{r-1}+1\right)^{2} \\
& <\prod_{j=1}^{L}\left(1+C_{m, 1} \nu^{-1} t_{j} T_{L}^{2}\right)-1 .
\end{aligned}
$$

We have proved Theorem 2 upto the proof of Lemma 5.1.

Proof of Lemma 5.1. We prove Lemma by induction on $s$. The case $s=1$. We abbreviate $j_{1}=j$. Just as in the proof of Lemma 4.2, we let

$$
\tilde{b}_{j}\left(x_{L}, \cdots, x_{j+1}, x_{j}, x_{0}\right)=R_{j-1} S_{j-2} \cdots S_{1}(1) .
$$

Then this is defined by the equality 


$$
\begin{gathered}
\left(\frac{E}{t_{j}}\right)^{1 / 2}\left(\frac{E}{T(j-1,1)}\right)^{1 / 2} \int_{\mathrm{R}} e^{-i_{\nu}\left(S_{j}\left(t_{j}, x_{j}, x_{j-1}\right)+s_{j-1,1}^{\#}\left(x_{j-1}, x_{0}\right)\right)} D\left(x_{j-1}, x_{0}\right)^{-1 / 2} d x_{j-1} \\
=\left(\frac{E}{T(i, 1)}\right)^{1 / 2} e^{-i_{\nu} S_{j, 1}^{\#}\left(x_{j}, x_{0}\right)}\left(D\left(x_{j}, x_{0}\right)^{-1 / 2}+\tilde{b}_{j}\left(x_{L}, \cdots, x_{j+1}, x_{j}, x_{0}\right)\right)
\end{gathered}
$$

This means that $\tilde{b}_{j}\left(x_{L}, \cdots, x_{j+1}, x_{j}, x_{0}\right)$ is indepenent of $x_{L}, \cdots, x_{j+1}$. We can write, $\tilde{b}_{j}\left(x_{L}, \cdots, x_{j+1}, x_{j}, x_{0}\right)$ by $\tilde{b}_{j}\left(x_{j}, x_{0}\right)$. Furthermore we wish to show that we can write

$$
\tilde{b}_{j}\left(x_{j}, x_{0}\right)=\nu^{-1} t_{j} T(j-1,1)^{2} p\left(x_{j}, x_{0}\right) .
$$

To show (5.14), we need a closer look at the amplitude function of (5.13). By virtue of Proposition 2.10, there exists a function $q_{j}\left(x_{j-1}, x_{0}\right) \in$ $\mathscr{B}(\mathbb{R} \times \mathbb{R})$ such that we have

$$
D\left(x_{j-1}, x_{0}\right)^{-1 / 2}=1+T(j-1,1)^{2} q_{j-1}\left(x_{j-1}, x_{0}\right) .
$$

This means that

$$
\partial_{x_{j-1}} D\left(x_{j-1}, x_{0}\right)^{-1 / 2}=T(j-1,1)^{2} \partial_{x_{j-1}} q_{j-1}\left(x_{j-1}, x_{0}\right) .
$$

We apply Lemma 3.5 to (5.13). For any $m$ there exists a constant $C_{m}$ such that if $\left|\alpha_{0}\right|,\left|\alpha_{j}\right| \leqq m$ we have

$$
\begin{aligned}
\left|\partial_{x_{j}}^{\alpha_{j}} \partial_{x_{0}}^{\alpha_{0}} \tilde{b}_{j}\left(x_{j}, x_{0}\right)\right| & \leqq C_{m}\left(\frac{t_{j} T(j-1,1)}{\nu T(j, 1)}\right) \max \sup _{x_{j-1}}\left|\partial_{x_{j-1}}^{\beta_{j-1}} \partial_{x_{0}}^{\beta_{0}}\left(D\left(x_{j-1}, x_{0}\right)^{-1 / 2}\right)\right| \\
& \leqq C_{m}\left(\frac{t_{j} T(j-1,1)^{3}}{\nu T(j, 1)}\right) \max \sup _{x_{j-1}}\left|\partial_{x_{j-1}}^{\beta_{j-1}} \partial_{x_{0}}^{\beta_{0}} q_{j-1}\left(x_{j-1}, x_{0}\right)\right|
\end{aligned}
$$

Here max is taken over $\beta_{j}$ and $\beta_{0}$ with $1 \leqq\left|\beta_{j-1}\right| \leqq 2 m+4+2,\left|\beta_{0}\right| \leqq m$. This proves (5.14). Lemma 5.1 for $s=1$ is true.

Assuming Lemma 5.1 for $s$, we prove it for $s+1$. By induction hypothesis the function $\tilde{b}_{j_{s} j_{s-1} \cdots j_{1}}\left(x_{L}, \cdots, x_{j_{s+1}}, x_{j_{s}}, x_{j_{s-1}}, \cdots, x_{j_{1}}, x_{0}\right)$ does not depend on $x_{L}, \cdots, x_{j_{s+1}}$. So we may denote it by

$$
\tilde{b}_{j_{s} j_{s-1} \cdots j_{1}}\left(x_{j_{s}}, x_{j_{s-1}}, \cdots, x_{j_{1}}, x_{0}\right) \text {. }
$$

Let $j_{s+1}$ be an arbitrary integer such that $j_{s}+1<j_{s+1}<L$. Then we have by definition (5.9).

$$
\tilde{b}_{j_{s+1} j_{s} \cdots j_{1}}=R_{j_{s+1}-1} S_{j_{s+1}-2} \cdots S_{j_{s+1}} \tilde{b}_{j_{s} j_{s-1} \cdots j_{1}} .
$$

We see that 


$$
\begin{aligned}
& S_{j_{s+1}-2} \cdots S_{j_{s+1}} \tilde{b}_{j_{s} j_{s-1} \ldots j_{1}}(1)\left(x_{L}, \cdots, x_{j_{s+1}-1}, x_{j_{s}}, \cdots, x_{j_{1}}, x_{0}\right) \\
& \quad=D\left(x_{j_{s+1}-1}, x_{j_{s}}\right)^{-1 / 2} \tilde{b}_{j_{s} j_{s-1} \ldots j_{1}}\left(x_{j_{s}}, x_{j_{s-1}}, \cdots, x_{j_{1}}, x_{0}\right) .
\end{aligned}
$$

Here we set $D\left(x_{j_{s+1}-1}, x_{j_{s}}\right)=1$ if $j_{s+1}-1=j_{s}+1$. Thus (5.17) and (5.18) imply that the function

$$
\tilde{b}_{j_{s+1} j_{s} \cdots j_{1}}\left(x_{L}, \cdots, x_{j_{s+1}+1}, x_{j_{s+1}}, x_{j_{s}}, \cdots, x_{j_{1}}, x_{0}\right)
$$

is defined by the equality:

$$
\begin{aligned}
& \left(\frac{E}{t_{j_{s+1}}}\right)^{1 / 2}\left(\frac{E}{T\left(j_{s+1}-1, j_{s}+1\right)}\right)^{1 / 2} \\
& \times \int_{\mathbf{R}} e^{-i \nu\left(S_{j_{s+1}}\left(t_{j_{s+1}}, x_{j_{s+1}}, x_{j_{s+1}-1}\right)+S_{j_{s+1}-1, j_{s}+1}^{\#}\left(x_{j_{s+1}-1}, x_{j_{s}}\right)\right)} \\
& \times D\left(x_{j_{s+1}-1}, x_{j_{s}}\right)^{-1 / 2} \tilde{b}_{j_{s} j_{s-1} \ldots j_{1}}\left(x_{j_{s}}, x_{j_{s-1}}, \cdots, x_{0}\right) d x_{j_{s+1}-1}
\end{aligned}
$$

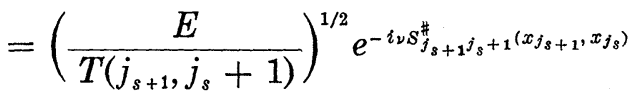

$$
\begin{aligned}
& \times\left(D\left(x_{j_{s+1}}, x_{j_{s}}\right)^{-1 / 2} \tilde{b}_{j_{s} j_{s-1} \ldots j_{1}}\left(x_{j_{s}}, x_{j_{s-1}}, \cdots, x_{0}\right)\right. \\
& \left.+\tilde{b}_{j_{s+1} j_{s} \cdots j_{1}}\left(x_{L}, \cdots, x_{j_{s+1}+1}, x_{j_{s+1}}, x_{j_{s}}, \ldots, x_{j_{1}}, x_{0}\right)\right) \text {. }
\end{aligned}
$$

The left hand side of this equals

$$
\begin{aligned}
& \tilde{b}_{j_{s} j_{s-1} \cdots i_{1}}\left(x_{j_{s}}, x_{j_{s-1}}, \cdots, x_{0}\right)\left(\frac{E}{t_{j_{s+1}}}\right)^{1 / 2}\left(\frac{E}{T\left(j_{s+1}-1, j_{s}+1\right)}\right)^{1 / 2} \\
& \quad \times \int_{\mathrm{R}} e^{-i_{\nu}\left(s_{j_{s+1}}\left(t_{j_{s+1}}, x_{j_{s+1}}, x_{j_{s+1}-1}\right)+s_{j_{s+1}-1, j_{s}+1}^{\sharp}\left(x_{j_{s+1}-1}, x_{f_{s}}\right)\right)} D\left(x_{j_{s+1}-1}, x_{j_{s}}\right)^{-1 / 2} d x_{j_{s+1}-1} .
\end{aligned}
$$

The last integral was treated earlier in (5.13). Using discussions there, we can prove that (5.19) equals

$$
\begin{aligned}
& \left(\frac{E}{T\left(j_{s+1} j_{s}+1\right)}\right)^{1 / 2} e^{-i \nu s_{j_{s+1}, j_{s}+1}^{\#}\left(x_{j_{s+1}}, x_{j_{s}}\right)} \tilde{b}_{j_{s} \ldots j_{1}}\left(x_{j_{s}}, \cdots, x_{0}\right) \\
& \quad \times\left(D\left(x_{j_{s+1}}, x_{j_{s}}\right)^{-1 / 2}+\nu^{-1} t_{j_{s+1}} T\left(j_{s+1}-1, j_{s}+1\right)^{2} p_{j_{s+1}}\left(x_{j_{s+1}}, x_{j_{s}}\right)\right)
\end{aligned}
$$

with some $p_{j_{s+1}}\left(x_{j_{s+1}}, x_{j_{s}}\right) \in \mathscr{B}(\mathbb{R} \times \mathbb{R})$. It follows from this that

$$
\begin{aligned}
& \tilde{b}_{j_{s+1} j_{s} \cdots j_{1}}\left(x_{L}, \cdots, x_{j_{s+1}}, x_{j_{s+1}}, x_{j_{s}}, \cdots, x_{j_{1}}, x_{0}\right) \\
& \quad=\left(\frac{t_{j_{s+1}} T\left(j_{s+1}-1, j_{s}+1\right)^{2}}{\nu}\right) p_{j_{s+1}}\left(x_{j_{s+1}}, x_{j_{s}}\right) \tilde{b}_{j_{s} \cdots j_{1}}\left(x_{j_{s}}, \cdots, x_{j_{1}}, x_{0}\right) .
\end{aligned}
$$

We can use induction hypothesis for $\tilde{b}_{j_{s} \ldots j_{1}}\left(x_{j_{s}}, \cdots, x_{j_{1}}, x_{0}\right)$, i.e., replace $\tilde{b}_{j_{s} \ldots j_{1}}\left(x_{j_{s}}, \cdots, x_{j_{1}}, x_{0}\right)$ in (5.20) by the right hand side of (5.10). Consequently, we obtain 


$$
\begin{aligned}
\tilde{b}_{j_{s+1} \ldots j_{1}}\left(x_{L}, \cdots, x_{j_{s+1}+1}, x_{j_{s+1}}, x_{j_{s}}, \cdots, x_{j_{1}}, x_{0}\right) & \\
=\left(\frac{t_{j_{s+1}} T\left(j_{s+1}-1, j_{s}+1\right)^{2}}{\nu}\right) & \prod_{r=1}^{s}\left(\frac{t_{j_{r}}}{\nu} T\left(j_{r}-1, j_{r-1}+1\right)^{2}\right) \\
& \times p_{j_{s+1}}\left(x_{j_{s+1}}, x_{j_{s}}\right) \prod_{r=1}^{s} p_{j_{r}}\left(x_{j_{r}}, x_{j_{r-1}}\right) .
\end{aligned}
$$

This proves (5.10) for $s+1$. Lemma 5.1 has been proved.

We have completed proof of Theorem 2 .

\section{REFERENCES}

[1] Asada, K.-Fujiwara, D., On some oscillatory integral transformations in $L^{2}\left(\mathbb{R}^{n}\right)$, Japan. J. Math., 4 (1978), 299-361.

[2] Feynman, R. P., Space time approach to non relativistic quantum mechanics, Rev. Modern Phys., 20 (1948), 367-387.

[3] Fujiwara, D., Remarks on convergence of some Feynman path integrals, Duke Math. J., 47 (1980), 559-600.

[4] - A remark on Taniguchi-Kumanogo theorem for product of Fourier integral operators, Pseudo-differential operators, Proc. Oberwolfach 1986, Lecture notes in Math. No. 1256, Springer (1987), 135-153.

[5] - - The Feynman path integral as an improper integral over the Sobolev space, Proc. of "Journee equations aux derivee partielles, Sain Jean de Monts 1990", Societe Math de France (1990), XIV 1-15.

[ 6 ] Kumanogo, H., Pseudo differential operators, MIT Press, (1982).

[ 7 ] Kumanogo, H.-Taniguchi, K., Fourier integral operators of multiphase and the fundamental solution for a hyperbolic system, Funkci. Ekvac., 22 (1979), pp. 161196.

[8] Taniguchi, K., Multi-products of Fourier integral operators and the fundamental solution for a hyperbolic system with involutive characteristics, Osaka J. Math., 21 (1984), 169-124.

Department of Mathematics

Tokyo Institute of Technology

2-12-1 Oh-okayama, Meguro-ku

Tokyo 152

Japan 